\title{
Valuations and Plurisubharmonic Singularities
}

Dedicated to Heisuke Hironaka on the occasion of his seventy-seventh birthday

By

Sébastien BoucKsom*, Charles FAvRE** and Mattias Jonsson***

\begin{abstract}
We extend to higher dimensions some of the valuative analysis of singularities of plurisubharmonic (psh) functions developed by the first two authors. Following Kontsevich and Soibelman we describe the geometry of the space $\mathcal{V}$ of all normalized valuations on $\mathbf{C}\left[x_{1}, \ldots, x_{n}\right]$ centered at the origin. It is a union of simplices naturally endowed with an affine structure. Using relative positivity properties of divisors living on modifications of $\mathbf{C}^{n}$ above the origin, we define formal psh functions on $\mathcal{V}$, designed to be analogues of the usual psh functions. For bounded formal psh functions on $\mathcal{V}$, we define a mixed Monge-Ampère operator which reflects the intersection theory of divisors above the origin of $\mathbf{C}^{n}$. This operator associates to any $(n-1)$-tuple of formal psh functions a positive measure of finite mass on $\mathcal{V}$. Next, we show that the collection of Lelong numbers of a given germ $u$ of a psh function at all infinitely near points induces a formal psh function $\hat{u}$ on $\mathcal{V}$. When $\varphi$ is a psh Hölder weight in the sense of Demailly, the generalized Lelong number $\nu_{\varphi}(u)$ equals the integral of $\hat{u}$ against the Monge-Ampère measure of $\hat{\varphi}$. In particular, any generalized Lelong number is an average of valuations. We also show how to compute the multiplier ideal of $u$ and the relative type of $u$ with respect to $\varphi$ in the sense of Rashkovskii, in terms of $\hat{u}$ and $\hat{\varphi}$.
\end{abstract}

Communicated by K. Saito. Received February 16, 2007.

2000 Mathematics Subject Classification(s): Primary 32U25; Secondary 13A18, 14B05, $32 \mathrm{P} 05$.

First author supported by the Japanese Society for the Promotion of Science. Third author supported by the NSF, the Swedish Research Council and the Gustafsson Foundation.

* CNRS-Université Paris 7, Institut de Mathématiques, F-75251 Paris Cedex 05, France. e-mail: boucksom@math.jussieu.fr

** CNRS-Université Paris 7, Institut de Mathématiques, F-75251 Paris Cedex 05, France and Unidade Mista CNRS-IMPA, Dona Castorina 110, Rio de Janeiro, 22460-320, Brazil. e-mail: favre@math.jussieu.fr

*** Dept. of Mathematics, KTH, SE-100 44 Stockholm, Sweden and Dept. of Mathematics, University of Michigan, Ann Arbor, MI 48109-1109, USA.

e-mail: mattiasj@umich.edu

(c) 2008 Research Institute for Mathematical Sciences, Kyoto University. All rights reserved. 


\section{Contents}

Introduction

$\S 1$. Geometry of Valuation Spaces

$\S 2$. Positivity and Convexity

$\S 3$. Valuative Multiplier Ideals

$\S 4$. Intersection Theory and Monge-Ampère Operator

$\S 5$. Singularities of Plurisubharmonic Functions

References

\section{Introduction}

This paper aims at laying down the foundations for a valuative study of singularities of plurisubharmonic (psh) functions in any dimension. Such an analysis was carried out by the last two authors in dimension two in a series of papers [FJ1], [FJ2], [FJ3]. But that work used in an essential way the assumption on the dimension, and did not immediately extend to a more general setting. Here we present a more geometric approach, using as a key tool Hironaka's theorem on resolution of singularities.

Fix $n \geq 1$ and let $u:\left(\mathbf{C}^{n}, 0\right) \rightarrow \mathbf{R} \cup\{-\infty\}$ be a psh germ, that is, a psh function defined in a neighborhood of the origin. We say that $u$ has a singularity at 0 when $u(0)=-\infty$. Various quantities have been introduced to measure the singularity of $u$ at 0 , the most classical certainly being the so-called Lelong number $\nu^{\mathrm{L}}(u, 0)=\max \{c>0, u(z) \leq c \log |z|+O(1)\}$. Although of fundamental importance, the Lelong number gives too rough an insight into the singularity of $u$ in many situations. To remedy this problem, we propose to look at the family of Lelong numbers $\nu^{\mathrm{L}}(u \circ \pi, p)$ at all points $p \in \pi^{-1}(0)$ lying in some birational model $\pi: X_{\pi} \rightarrow X:=\mathbf{C}^{n}$. The general aim of this article is to show that given this collection of Lelong numbers, one can recover essentially all other known measurements of singularity of $u$, including multiplier ideals, generalized Lelong numbers and relative types.

Theorem A. Let $u, v:\left(\mathbf{C}^{n}, 0\right) \rightarrow \mathbf{R} \cup\{-\infty\}$ be two psh germs. Consider the following four statements.

(1) For all proper modifications $\pi: X_{\pi} \rightarrow \mathbf{C}^{n}$ above 0 and all points $p \in \pi^{-1}(0)$, we have $\nu^{\mathrm{L}}(u \circ \pi, p)=\nu^{\mathrm{L}}(v \circ \pi, p)$. 
(2) For all $t>0$ we have $\mathcal{J}_{+}(t u)=\mathcal{J}_{+}(t v)$.

(3) For any tame maximal psh weight $\varphi$, the relative types $\sigma(u, \varphi)=\sup \{c>$ $0, u \leq c \varphi+O(1)\}$ and $\sigma(v, \varphi)$ are equal.

(4) For any tame psh weight $\varphi$, the generalized Lelong numbers $\nu_{\varphi}(u)=d d^{c} u \wedge$ $\left(d d^{c} \varphi\right)^{n-1}\{0\}$ and $\nu_{\varphi}(v)$ are equal.

Then (1), (2) and (3) are equivalent, and imply (4).

Let us explain the notation used in the statement of the theorem. For any psh germ $u$, we denote by $\mathcal{J}(u)$ its multiplier ideal, i.e. the ideal of germs of holomorphic functions $f$ at 0 such that $f e^{-u} \in L_{\text {loc }}^{2}$. In $(2) \mathcal{J}_{+}(u)$ refers to the stationary value of $\mathcal{J}((1+\varepsilon) u)$ as $\varepsilon>0$ tends to 0 .

A psh weight is a psh germ $\varphi$ with an isolated singularity at the origin such that $e^{\varphi}$ is continuous. A psh weight is said to be tame if it can be strongly approximated by its multiplier ideals, cf. Definition 5.8. In particular psh weights $\varphi$ such that $e^{\varphi}$ is Hölder continuous are tame. Finally, a weight is said to be maximal if it is locally maximal outside the origin, i.e. its MongeAmpère measure is a Dirac mass at 0 . The notion of generalized Lelong number is due to Demailly [D1], whereas the relative type was introduced more recently by Rashkovskii in [Ras1]. The theorem above shows that these notions can be read off directly from the Lelong numbers of $u$ in all birational models. We expect (4) to imply in turn the other statements and that $\mathcal{J}_{+}$can be replaced by $\mathcal{J}$ itself in (2) but further analysis is required to prove this.

At the heart of our method lies the idea of organizing the data $\left\{\nu^{\mathrm{L}}(u, p)\right\}$ in an efficient way, and turning it into a function on a suitable space of valuations. We shall prove that both this function and this space exhibit striking properties which relate them in a natural way to convex geometry. This feature will eventually help us to control the behavior of the Lelong numbers $\nu^{\mathrm{L}}(u \circ \pi, p)$ when moving the point $p$ in the tower of all blow-ups $\pi$.

To motivate the introduction of valuations, recall that for any holomorphic function $f$, the integer $\nu^{\mathrm{L}}(\log |f|)$ equals the multiplicity of $f$ at 0 . More generally, for any modification $\pi: X_{\pi} \rightarrow \mathbf{C}^{n}$, and any point $p \in \pi^{-1}(0)$, the function $f \mapsto \nu^{\mathrm{L}}(\log |f \circ \pi|, p)$ is equal to the divisorial valuation $f \mapsto \nu_{p}(f):=$ $\operatorname{ord}_{p}(f \circ \pi)$, defined on the $\operatorname{ring} R:=\mathbf{C}\left[x_{1}, \ldots, x_{n}\right]$. If $\mathcal{V}_{\text {div }}$ denotes the space of all (suitable normalized) divisorial valuations $\mathcal{V}_{\text {div }}$ centered at 0 , we see that the collection $\left\{\nu^{\mathrm{L}}(u \circ \pi, p)\right\}$ induces a real-valued function on $\mathcal{V}_{\text {div }}$. As we shall 
soon explain, this function, which will be denoted by $-\hat{u}$, is of a very special type.

Unfortunately, the natural topology of pointwise convergence turns $\mathcal{V}_{\text {div }}$ into a totally discontinuous space, and it is difficult to directly study functions on it. One is led to complete this space in some way. As it turns out, there are two reasonable choices that one can make, which we refer to as Zariski's and Berkovich's point of view. As we shall see, both approaches shed their own light onto the problem, and benefit one from another.

In Zariski's perspective [Z], one considers $\mathfrak{X}:=\lim _{\pi} X_{\pi}$ the projective limit over all proper modifications of $\pi: X_{\pi} \rightarrow X:=\mathbb{C}^{n}$ above 0 . This space contains all rank 1 valuation rings of the function field $\mathbf{C}(X)$ whose maximal ideal contain the maximal ideal $\mathfrak{m}$ of $X$ at 0 . We shall refer to it as the RiemannZariski space of $X$ at 0 . It clearly contains $\mathcal{V}_{\text {div }}$, and admits a natural topology for which it is quasi-compact. In $\mathfrak{X}$, divisors are in one-to-one correspondence with functions on the subspace $\mathcal{V}_{\text {div }} \subset \mathfrak{X}$, hence any psh germ defines a divisor $Z(u)$ on $\mathfrak{X}$.

The Berkovich point of view refers to the theory of analytic spaces over non-archimedean fields as developed in [Be]. In our context, we view $\mathbf{C}$ as a field with a trivial valuation, and define $\mathcal{V}$ to be the set of all valuations $\nu: R \rightarrow[0,+\infty]$ that extend this trivial valuation on $\mathbf{C}$, are $>0$ on the maximal ideal $\mathfrak{m}=\left(x_{1}, \ldots, x_{n}\right) \subset R$ and normalized by $\nu(\mathfrak{m}):=\min \nu\left(x_{i}\right)=1$. From this perspective, $\mathcal{V}_{\text {div }}$ becomes a dense subset of $\mathcal{V}$, which is compact for the topology of pointwise convergence. Of course, given a psh germ $u$, it is unclear whether the function $\hat{u}$, which is a priori defined only on $\mathcal{V}_{\text {div }}$ extends in a natural way to $\mathcal{V}$. But we may remark that in the case $u=\log |f|$ for some $f \in R$, the function $\hat{u}(\nu):=-\nu(f)$ is a natural choice, as it yields a continuous function on $\mathcal{V}$. We shall prove that for any psh germ $u$, the function $\hat{u}$ does indeed extend to $\mathcal{V}$ in a unique way with an appropriate continuity property.

To go further, it is now necessary to take a closer look at the space of divisors on $\mathfrak{X}$, and at the geometry of $\mathcal{V}$. Let us begin with the latter, following $[\mathrm{KKMS}]$ and $[\mathrm{KS}]$.

If $\pi: X_{\pi} \rightarrow \mathbf{C}^{n}$ is a modification above the origin with simple normal crossing exceptional divisor, then one can construct the dual (simplicial) complex $\Delta(\pi)$ of $\pi$ which encodes the incidence relations between the irreducible components of $\pi^{-1}(0)$. Its underlying topological space is a union of simplices of real dimension $\leq n-1$, and is homotopic to a point. What makes this 
construction useful is the fact that vertices of $\Delta(\pi)$ are irreducible components of $\pi^{-1}(0)$, and can thus be viewed as divisorial valuations included in $\mathcal{V}$. Moreover, the faces of $\Delta(\pi)$ can be naturally identified with valuations that are monomial in suitable local coordinates in $X_{\pi}$. Thus we can realize the underlying topological space of $\Delta(\pi)$ as a subset $|\Delta(\pi)|$ of $\mathcal{V}$. When $\pi^{\prime}$ dominates $\pi$, i.e. when there exists a modification $\mu: X_{\pi^{\prime}} \rightarrow X_{\pi}$ such that $\pi^{\prime}=\pi \circ \mu$, we have a natural inclusion $|\Delta(\pi)| \subset\left|\Delta\left(\pi^{\prime}\right)\right|$. Letting $\pi$ tend to infinity in the net of all modifications yields a dense inclusion from $\cup_{\pi}|\Delta(\pi)|$ into $\mathcal{V}$.

Being identified with sets of monomial valuations, faces of dual complexes are naturally parameterized by the weights on the corresponding coordinates in which the valuations are monomial and thus inherit an affine structure. A simplex in $\mathcal{V}$ is a face of some $|\Delta(\pi)|$ endowed with this affine structure. The affine structure on $\mathcal{V}$ is of fundamental importance.

Returning to a psh germ $u$, it is not difficult to interpret the restriction of $\hat{u}$ to a fixed simplex in terms of a weighted version of Lelong numbers called Kiselman numbers [Ki]. Using this fact, we conclude that the restriction of $\hat{u}$ to any simplex is convex. This is already a quite remarkable fact, but it is also of very local nature as simplices are relatively small subsets of $\mathcal{V}$. One may thus wonder if the function $\hat{u}$ does not exhibit more global convexity properties. Unfortunately it is not immediately clear how all the simplices patch together to build up $\mathcal{V},{ }^{1}$ so it seems hard to define any notion of convexity in $\mathcal{V}$ starting from the simplices. To overcome this difficulty, we need to turn our attention to Zariski's approach, describe the space of divisors on $\mathfrak{X}$ and study positivity properties on this space in the spirit of what was done in [BFJ].

A Weil divisor $Z$ on $\mathfrak{X}$ is defined as a collection $\left\{Z_{\pi}\right\}$ of divisors in all modifications $X_{\pi} \rightarrow X$ above 0, compatible under push-forward. Cartier divisors are those that are determined at a finite level $X_{\pi}$, that is, $Z_{\pi^{\prime}}=\mu^{*} Z_{\pi}$ for any $\mu: X_{\pi^{\prime}} \rightarrow X_{\pi}$. Clearly, a Weil divisor $Z$ is the same as a function $g_{Z}$ on $\mathcal{V}_{\text {div }}$, and it is not difficult to see that when $Z$ is Cartier, $g_{Z}$ extends as a continuous function on $\mathcal{V}$. The $\operatorname{space} \mathrm{C}-\operatorname{Div}(\mathfrak{X})$ of Cartier divisors sits as a dense subspace inside the space $\operatorname{Div}(\mathfrak{X})$ of Weil divisors, and both spaces are infinite dimensional.

Now any psh germ $u$ induces a Weil divisor $Z(u)$ given in $X_{\pi}$ by the Siu decomposition $d d^{c}(u \circ \pi)=-\left[Z(u)_{\pi}\right]+T_{\pi}^{\prime}$ with $T_{\pi}^{\prime}$ a positive closed $(1,1)$ current not charging $\pi^{-1}(0)$.

A closer look reveals that the divisor $Z(u)$ possesses a quite strong numerical positivity property: it lies in the closure of the cone of nef Cartier divisors,

\footnotetext{
${ }^{1}$ Except when $n=2$ where simplices are segments, and $\mathcal{V}$ is a real tree, see [FJ1].
} 
i.e. those Cartier divisors on $\mathfrak{X}$ that are determined by a nef divisor on some $X_{\pi}$. A Weil divisor in this closure will be said to be nef. We shall prove that for any nef Weil divisor $Z$ the corresponding function $g_{Z}$ on $\mathcal{V}_{\text {div }}$ extends to $\mathcal{V}$ in a natural way, see Section 3.4.

Using this notion of nef Weil divisor, we finally arrive at the global convexity notion on $\mathcal{V}$ that we were seeking. Namely, we define a formal psh function to be an upper semicontinuous function $g: \mathcal{V} \rightarrow[-\infty, 0]$ that is continuous on each simplex and whose restriction to $\mathcal{V}_{\text {div }}$ defines a nef Weil divisor. A formal psh function is convex on each simplex in $\mathcal{V}$, but the global convexity property recasts informations on the differential of the function when passing from one simplex to another.

As we shall see, formal psh functions on $\mathcal{V}$ have many properties in common with psh germs in $\mathbf{C}^{n}$. By construction, any function $\hat{u}$ associated to a psh germ is formal psh on $\mathcal{V}$. We call it the valuative transform of $u$.

In the philosophy underlying [BGS], one might want to think of a nef Weil divisor $Z$ as the formal closed, positive $(1,1)$-current $i \partial \bar{\partial} g_{Z}$ associated to the formal psh function $g_{Z}$.

We have thus associated to a psh germ $u$ on $\left(\mathbf{C}^{n}, 0\right)$ a nef Weil divisor $Z(u)$ on $\mathfrak{X}$ and a formal psh function $\hat{u}$ on $\mathcal{V}$. They encode the same information but from two different perspectives. Let us now indicate how one can use these notions to actually prove Theorem A.

Condition (1) says that two psh germs $u$ and $v$ have the same associated valuative transforms: $\hat{u}=\hat{v}$. Thus the equivalence between (1) and (2) follows from the understanding of the connection between $\hat{u}$ and the multiplier ideals of $u$. We put this problem into a larger perspective and define for any formal psh function $g$ on $\mathcal{V}$ a valuative multiplier ideal denoted by $\mathcal{L}^{2}(g)$, which is an ideal of the ring $\hat{R}=\mathbf{C}\left[\left[x_{1}, \ldots, x_{n}\right]\right]$ of formal power series. Although we do not know ${ }^{2}$ if the valuative multiplier ideal $\mathcal{L}^{2}(\hat{u})$ coincides with the usual multiplier ideal $\mathcal{J}(u)$ in the ring $\mathcal{O}$ of holomorphic germs at 0 , we can prove that $\mathcal{L}^{2}(\hat{u}) \cap \mathcal{O}=\mathcal{J}_{+}(u)$, and this shows that (1) implies (2). Conversely, Demailly's approximation results show that the singularities of a psh germ $u$ can be approximated by its multipliers ideals $\mathcal{J}(k u)$ as $k \rightarrow \infty$ in a very precise way, and we show that this is enough to recover $\hat{u}$, extending the analysis of [FJ3]. Thus (2) implies (1).

That (1) implies (3) is a consequence of the following result: if $\varphi$ is a tame

\footnotetext{
${ }^{2}$ If true, this would prove the Demailly-Kollár Openness Conjecture, see below.
} 
psh weight, then a psh germ $u$ satisfies $u \leq \varphi+O(1)$ iff $\hat{u} \leq \hat{\varphi}$. This is proved by another application of Demailly's results. Conversely, we show that (3) implies (1) by showing that quasi-monomial valuations can be represented as relative types with respect to tame psh weights.

To prove that (1) implies (4) we compute the mass of the Monge-Ampère measure $d d^{c} u \wedge\left(d d^{c} \varphi\right)^{n-1}$ at the origin in terms of the formal psh functions $\hat{u}$ and $\hat{\varphi}$ (with $\varphi$ a psh weight). This is done by introducing an intersection theory on nef divisors on $\mathfrak{X}$ and transferring its outcome to $\mathcal{V}$. More precisely, we proceed as follows.

The intersection of $n$ divisors in a fixed model $X_{\pi}$ leads to the definition of the intersection product $\left\langle Z_{1}, \ldots, Z_{n}\right\rangle$ of $n$ Cartier divisors. Using a monotonicity property of this product with respect to nef divisors, we extend the definition to arbitrary nef Weil divisors by approximation, and give a meaning to the intersection $\left\langle Z, Z_{2}, \ldots, Z_{n}\right\rangle$ of a Cartier divisor $Z$ against nef Weil divisors. We then show that the linear map $g_{Z} \mapsto\left\langle Z, Z_{2}, \ldots, Z_{n}\right\rangle \in \mathbf{R}$ extends to a positive linear continuous functional on all continuous functions on $\mathcal{V}$. This functional is thus attached to a positive Radon measure, which we call the Monge-Ampère measure of the formal psh functions $g_{i}$ associated to the nef Weil divisors $Z_{i}$. We denote it by $\operatorname{MA}\left(g_{1}, \ldots, g_{n-1}\right)$. As a measure on the $(n-1)$-dimensional space $\mathcal{V}$ it formally possesses many features of its complex analytic analog $d d^{c} u_{1} \wedge \cdots \wedge d d^{c} u_{n}$ on $\mathbf{C}^{n}$. The statement (1) $\Rightarrow$ (4) reflects a deeper connection between these two theories.

Theorem B. For any tame psh weight $\varphi$ and any psh germ $u$, the formal psh function $\hat{u}$ is integrable with respect to the positive Radon measure $\operatorname{MA}(\hat{\varphi})$, and

$$
\nu_{\varphi}(u):=\left(d d^{c} u \wedge\left(d d^{c} \varphi\right)^{n-1}\right)\{0\}=\int_{\mathcal{V}}-\hat{u} \operatorname{MA}(\hat{\varphi}) .
$$

In particular, the generalized Lelong number $\nu_{\varphi}$ is an average of valuations.

Here $\operatorname{MA}(\hat{\varphi})$ is a shorthand for $\operatorname{MA}(\hat{\varphi}, \ldots, \hat{\varphi})$. Technically the proof relies on yet another application of Demailly's approximation technique. A toric version of this result has been proved by Rashkovskii [Ras2].

The present work can be seen as an instance of developing a (pluri)potential theory in a non-Archimedean setting. Our method relies in an essential way on vanishing theorems available only in (residual) characteristic zero. But an 
extensive literature is devoted to such a goal over $p$-adic fields in connection with problems from arithmetic geometry see e.g. [A], [Bo], [CLR], [G], [K], [M], [Zh]. A potential theory for analytic curves defined over arbitrary non-Archimedean fields has been developed by Thuillier [T1], see also [BR], [FJ1]. On any analytic variety of any dimension, a notion of current and form have been introduced by Bloch-Gillet-Soulé [BGS], and measures coming from intersection of cycles have been studied by Chambert-Loir in [Ch].

There are several questions left open by the present work, notably the remaining implication $(4) \Longrightarrow(1)$ in Theorem A. One way to prove this implication would be to find fundamental solutions to the formal Monge-Ampère operator. Another interesting question is the Openness Conjecture by Demailly and Kollár [DK, Remark 5.3]: is $\mathcal{J}_{+}(u)=\mathcal{J}(u)$ for any psh germ $u$ ? The technique used in [FJ3] to prove this conjecture in dimension two relies on a more detailed understanding on the behavior of formal psh functions. It would also be of interest to generalize the dynamical results from [FJ4] to higher dimensions. We plan to come back to these issues in future work.

Organization of the paper. Section 1 contains basic definitions concerning the space of divisors in $\mathfrak{X}$, and a thorough discussion of the geometry of $\mathcal{V}$, mostly borrowed from [KKMS] and [KS] with a few adaptations to our local context. In Section 2, we undertake the study of positivity properties of divisors in $\mathfrak{X}$ and transport them to $\mathcal{V}$ with its affine structure. Section 3 contains a general discussion of valuative multiplier ideals. These are used to approximate formal psh functions by ideals, a powerful technique that plays a fundamental role in the definition of the Monge-Ampère operator, see Remark 4.5. The latter operator is introduced and discussed in Section 4. Finally we prove Theorems A and B in Section 5.

Setting. In Sections 1 to 4 , we let $k$ be an algebraically closed field of characteristic 0 and choose $0 \in X$ as a smooth closed point in an $n$-dimensional affine $k$-variety, with coordinate ring $R$ and maximal ideal $\mathfrak{m}$ at 0 . The formal completion of $X$ at 0 will be denoted by $\hat{X}$. It is the infinitesimal neighborhood of 0 in $X$, and is described by its coordinate ring $\hat{R}$, the completion of $R$ in the $\mathfrak{m}$-adic topology. Since 0 is a smooth point, $\hat{R}$ is isomorphic to $k\left[\left[x_{1}, \ldots, x_{n}\right]\right]$ for any choice of a coordinate system $x_{1}, \ldots, x_{n}$ at 0 .

In Section 5 where we speak about psh functions, we shall return to the complex setting $k=\mathbf{C}$, with 0 being the origin in $X=\mathbf{C}^{n}$. 
The following table might help the reader in translating notions from the geometric (or Zariski) point of view to the functional (or Berkovich) one.

\begin{tabular}{|l|l|}
\hline Berkovich point of view (functional) & Zariski point of view (geometric) \\
\hline Valuative space $\mathcal{V} \S 1.3$ & Riemann-Zariski space $\mathfrak{X} \S 1.1$ \\
Functions on $\mathcal{V}_{\text {div }}$ & Weil divisors $\S 1.2$ \\
Formal psh functions $\S 2.1$ & Nef Weil divisors $\S 2.2$ \\
Monge-Ampère operator $\S 4.2$ & Intersection theory of nef Weil divisors $\S 4.1$ \\
\hline
\end{tabular}

\section{$\S 1$. Geometry of Valuation Spaces}

\section{$\S 1.1$. The Riemann-Zariski space}

This space was introduced by Zariski [Z] as a tool for desingularizing algebraic varieties, see $[\mathrm{ZS}],[\mathrm{V}],[\mathrm{Co}]$ for a more detailed account on its definition and its history. The ringed space structure on the Riemann-Zariski space was introduced by Hironaka in [Hi].

Denote by $\mathfrak{B}$ the set of all projective modifications $\pi: X_{\pi} \rightarrow X$ above the origin (i.e. $\pi$ is an isomorphism above $X \backslash\{0\}$ ) such that $X_{\pi}$ is smooth. If $\pi, \pi^{\prime} \in \mathfrak{B}$, we say that $\pi^{\prime}$ dominates $\pi$ and write $\pi^{\prime} \geq \pi$, if there exists a morphism (necessarily unique and birational) $\mu: X_{\pi^{\prime}} \rightarrow X_{\pi}$ such that $\pi^{\prime}=\pi \circ$ $\mu$. This endows $\mathfrak{B}$ with a partial order relation. By Hironaka's desingularization theorem, this ordered set forms a directed family. The Riemann-Zariski space of $X$ at 0 is the projective limit of locally ringed topological spaces

$$
\mathfrak{X}:=\lim _{\pi} X_{\pi},
$$

where $X_{\pi}$ is viewed as a scheme. It can be shown that the locally ringed space $\mathfrak{X}$ is not isomorphic to a scheme, even of infinite type.

Remark 1.1. A comment on our terminology is in order. In dimension 2 , the space $\mathfrak{X}$ coincides with the set $\hat{\mathfrak{X}}$ of all valuation rings containing $\mathfrak{m}$, but this fact is no longer true as soon as $n \geq 3$. There are however natural maps $\imath: \mathfrak{X} \hookrightarrow \hat{\mathfrak{X}}$, and $p: \hat{\mathfrak{X}} \rightarrow \mathfrak{X}$ such that $\imath$ is injective, $p$ is surjective and $p \circ \imath$ is the identity on $\mathfrak{X}$. The map $p$ is defined by sending a valuation to the family of its centers in all models $X_{\pi}$ with $\pi \in \mathfrak{B}$. Note that Zariski's theorem says that a valuation is determined by the family of its centers in all birational models, not only those which are isomorphic outside the origin. For a point $x=\left\{x_{\pi}\right\} \in \mathfrak{X}$, let $\mathcal{I}_{x}$ be the prime ideal of those functions $f \in R$ for which $x_{\pi}$ is included in the strict transform of $f=0$ in $X_{\pi}$ for all $\pi$. When $\mathcal{I}_{x}=(0)$ the union of all local rings $\mathcal{O}_{x_{\pi}}$ is a valuation ring which we define to be $\imath(x)$. Otherwise, define 
$C_{x, 1}=\operatorname{Spec} \mathcal{O} / \mathcal{I}_{x}$. The collection $\left\{x_{\pi}\right\}$ now defines a point in the projective limit of the strict transforms of $C_{x, 1}$ by all $\pi$. We can thus inductively define a nested sequence of irreducible subschemes $C_{x, 1} \supsetneq C_{x, 2} \supsetneq \cdots C_{x, k}$. On $\mathcal{O}_{C_{x, k}}$ the point $x$ defines a valuation ring $\nu$. Set $\imath(x)$ to be the composed valuations of the divisorial valuations $\operatorname{ord}_{C_{x, j}}$ for $j=1, \ldots, k-1$ together with $\nu$. From this description of $\imath$ and $p$, one sees that $\mathfrak{X}$ contains all rank 1 valuations centered at 0 .

\section{$\S 1.2$. Divisors on $\mathfrak{X}$}

On the projective limit space $\mathfrak{X}$, one can define in a natural way Weil and Cartier divisors as limits of Weil and Cartier divisors on the $X_{\pi}$ 's. This is basically Shokurov's notion of birational divisors (or b-divisors) on $X$, cf. [S]. The subsequent discussion is adapted from [BFJ].

For $\pi \in \mathfrak{B}$, we write $\operatorname{Div}(\pi)$ for the set of divisors on $X_{\pi}$ with real coefficients and exceptional for $\pi$. It is a finite dimensional real vector space endowed with its canonical topology. Note that an exceptional divisor is uniquely determined by its numerical class, so that we obtain a natural isomorphism between $\operatorname{Div}(\pi)$ and the relative Néron-Severi space $N S\left(X_{\pi} / X\right)_{\mathbf{R}}$ of $X_{\pi}$ (recall that $X_{\pi}$ is assumed to be smooth). A birational morphism $\mu: X_{\pi^{\prime}} \rightarrow X_{\pi}$ with $\pi^{\prime}, \pi \in \mathfrak{B}$ induces natural linear maps $\mu^{*}: \operatorname{Div}(\pi) \rightarrow \operatorname{Div}\left(\pi^{\prime}\right), \mu_{*}: \operatorname{Div}\left(\pi^{\prime}\right) \rightarrow \operatorname{Div}(\pi)$.

\section{Definition 1.2.}

- The space of Weil divisors on $\mathfrak{X}$ is the projective limit

$$
\operatorname{Div}(\mathfrak{X}):=\varliminf_{\pi}^{\lim } \operatorname{Div}(\pi)
$$

with respect to the push-forward arrows. It is endowed with its projective limit topology, which will be called the weak topology.

- The space of Cartier divisors on $\mathfrak{X}$ is the inductive limit

$$
\mathrm{C}-\operatorname{Div}(\mathfrak{X}):=\underset{\pi}{\lim } \operatorname{Div}(\pi)
$$

with respect to the pull-back arrows. It is endowed with its inductive limit topology, which will be called the strong topology.

Note that these vector spaces are infinite dimensional since we assume $n \geq 2$. We will denote by $\operatorname{Div}(\mathfrak{X})_{\mathbf{Z}}$ and $\operatorname{C-Div}(\mathfrak{X})_{\mathbf{Z}}$ the spaces of Weil and Cartier divisors with $\mathbf{Z}$-coefficients. 
A Weil divisor $Z \in \operatorname{Div}(\mathfrak{X})$ is by definition described by its incarnations $Z_{\pi} \in \operatorname{Div}(\pi)$ on each smooth birational model $X_{\pi}$ of $X$, compatible with each other by push-forward. A sequence (or a net) $Z_{i}$ converges to $Z$ in the weak topology iff $Z_{i, \pi} \rightarrow Z_{\pi}$ in $\operatorname{Div}(\pi)$ for each $\pi$.

On the other hand, the relation $\mu_{*} \mu^{*} \alpha=\alpha$ when $\mu$ is a birational morphism shows that there is an injection

$$
\operatorname{C-Div}(\mathfrak{X}) \hookrightarrow \operatorname{Div}(\mathfrak{X})
$$

i.e. a Cartier divisor is in particular a Weil divisor. Concretely, a Weil divisor $Z$ is Cartier iff there exists $\pi$ such that its incarnations $Z_{\pi^{\prime}}$ on higher blow-ups $X_{\pi^{\prime}}$ are obtained by pulling-back $Z_{\pi}$. We will call such a $\pi$ a determination of $Z$. There are also natural injective maps

$$
\operatorname{Div}(\pi) \hookrightarrow \operatorname{C}-\operatorname{Div}(\mathfrak{X})
$$

which extend a given divisor $Z \in \operatorname{Div}(\pi)$ to a Cartier divisor by pulling it back. This Cartier divisor is by construction determined by $\pi$. In the sequel, we shall always identify a divisor $Z \in \operatorname{Div}(\pi)$ with its image in $\mathrm{C}$-Div $(\mathfrak{X})$.

A function on $\mathrm{C}-\operatorname{Div}(\mathfrak{X})$ is continuous in the strong topology iff its restriction to each $\operatorname{Div}(\pi)$ is continuous. In particular, the natural injective map $\mathrm{C}-\operatorname{Div}(\mathfrak{X}) \rightarrow \operatorname{Div}(\mathfrak{X})$ is continuous, and moreover has dense image. Indeed, if $Z$ is a Weil divisor on $\mathfrak{X}$, then the incarnations $Z_{\pi}$ of $Z$ on $X_{\pi}$, viewed as Cartier (hence Weil) divisors on $\mathfrak{X}$, tautologically converge to $W$ as $\pi \rightarrow \infty$.

If $\mathfrak{a} \subset \hat{R}$ is an ideal, we let $Z(\mathfrak{a})$ be the Weil divisor on $\mathfrak{X}$ such that $\operatorname{ord}_{E} Z(\mathfrak{a})=-\operatorname{ord}_{E}(\mathfrak{a})$ for each exceptional prime $E$. Note that $Z(\mathfrak{a}) \leq 0$. When $\mathfrak{a}=(f)$ is principal, we also write $Z(f)$. The divisor $Z(\mathfrak{a})$ on $\mathfrak{X}$ is Cartier iff $\mathfrak{a}$ is a primary ideal. By the Nullstellensatz, a primary ideal $\mathfrak{a}$ of $\hat{R}$ is generated by $\mathfrak{a} \cap R$, so that primary ideals in $R$ and $\hat{R}$ coincide. In that case, $Z(\mathfrak{a})$ is the Cartier divisor such that $-Z(\mathfrak{a})_{\pi}$ is the effective Cartier divisor defined by the principal ideal sheaf $\pi^{-1} \mathfrak{a}$ whenever $\pi$ dominates the blow-up of $\mathfrak{a}$.

\section{$\S 1.3$. Valuations}

By a valuation $\nu$ on $X=\operatorname{Spec}(R)$, we mean a function $\nu: R \rightarrow[0,+\infty]$ such that $\nu\left(f_{1} f_{2}\right)=\nu\left(f_{1}\right)+\nu\left(f_{2}\right), \nu\left(f_{1}+f_{2}\right) \geq \min \left\{\nu\left(f_{1}\right), \nu\left(f_{2}\right)\right\}$ for all $f_{1}, f_{2} \in$ $R$, and which induces the trivial valuation on $k \subset R$, i.e. $\nu(0)=+\infty,\left.\nu\right|_{k^{*}} \equiv 0$, and satisfying $\nu(\mathfrak{m})>0$. This is the same as a non-Archimedean absolute value $\exp (-\nu)$ on $R$ that restricts to the trivial one on $k$. We will denote by 
$f \mapsto|f|(\nu):=\exp (-\nu(f))$, or simply $f \mapsto|f|$, the corresponding absolute value, following standard notation in non-Archimedean geometry.

The ideal $\nu^{-1}(+\infty)$ is a prime ideal, cutting out a subvariety $H=H(\nu, X)$ of $X$ called the home of $\nu$ in $X$. Note that $\nu$ induces a real-valued valuation in the sense of [ZS, §8] on the function field $k(H)$ of its home, so we can attach to $\left.\nu\right|_{H}$ the usual invariants of valuation theory. In particular, we can consider the value group $\Gamma_{\nu}:=\nu\left(k(H)^{*}\right) \subset \mathbf{R}$ and residue field $\kappa_{\nu}:=\{\nu \geq 0\} /\{\nu>0\}$. Then the Abhyankar inequality holds:

$$
\text { tr. } \operatorname{deg}_{k}\left(\kappa_{\nu}\right)+\operatorname{dim}_{\mathbf{Q}}\left(\Gamma_{\nu} \otimes \mathbf{z} \mathbf{Q}\right) \leq \operatorname{dim} H(\nu, X) .
$$

The center of a valuation $\nu$ on $X$ is the subvariety of $H$ defined by the prime ideal $\{\nu>0\} \subset R$.

There exists a unique valuation whose home is reduced to 0 , namely the trivial valuation $\nu_{0}$ sending all elements of $\mathfrak{m}$ to $+\infty$. Now pick any non-trivial valuation $\nu$. For any projective modification $\pi: X_{\pi} \rightarrow X$ of $X$ above $0, \nu$ induces a valuation on $X_{\pi}$, i.e. a collection of compatible valuations on the local rings of $X_{\pi}$. This is clear when the home of $\nu$ is $X$ itself since $X$ and $X_{\pi}$ have the same function fields. And the general case reduces to this one: the home of $\nu$ on $X_{\pi}$ is then the strict transform of its home on $X$. In particular, we can consider the center of $\nu$ on $X_{\pi}$ : it is an irreducible subvariety.

If $\mathfrak{a}$ is an ideal of $R$, we set $\nu(\mathfrak{a}):=\min \{\nu(f), f \in \mathfrak{a}\}$, so that $\log |\mathfrak{a}|(\nu)=$ $-\nu(\mathfrak{a})$. A valuation $\nu$ on $R$ is centered at 0 iff $\nu(\mathfrak{m})>0$, which holds iff the corresponding absolute value $|\cdot|(\nu)$ continuously extends to an absolute value on $\hat{R}$ with its $\mathfrak{m}$-adic topology. Thus the set of valuations centered at 0 on $X$ can be identified with the set of continuous valuations on $\hat{R}$. Any non-trivial valuation $\nu$ on $X$ centered at 0 satisfies $0<\nu(\mathfrak{m})<+\infty$, so that we can normalize it as follows.

Definition 1.3. We denote by $\hat{\mathcal{V}}$ the set of all valuations $\nu$ on $X$ centered at 0 , and by $\mathcal{V}$ the subspace of those valuations normalized by $\nu(\mathfrak{m})=1$. We endow $\hat{\mathcal{V}}$ and $\mathcal{V}$ with the topology of pointwise convergence on $R$.

Then $\mathcal{V}$ is compact, and $\hat{\mathcal{V}}$ is endowed with a $\mathbf{R}_{+}^{*}$-action by scaling. The trivial valuation $\nu_{0}$ is the unique fixed point, and for each $\nu \in \hat{\mathcal{V}}$ we have $t \nu \rightarrow \nu_{0}$ as $t \rightarrow+\infty$. The space $\hat{\mathcal{V}} \backslash\left\{\nu_{0}\right\}$ can be identified with the open cone over $\mathcal{V}$. We will say that a function $g$ on $\hat{\mathcal{V}} \rightarrow[-\infty,+\infty]$ is homogeneous if it satisfies $g(t \nu)=t g(\nu)$ for each $t>0$ and each $\nu \in \hat{\mathcal{V}}$.

Remark 1.4. The space $\hat{\mathcal{V}}$ we consider here is by definition the $k$-analytic space in the sense of Berkovich associated by Thuillier in [T2] to the formal 
completion $\hat{X}$ of the $k$-scheme $X$ at the origin. The singleton $\left\{\nu_{0}\right\}$ is the $k$ analytic space associated to the point space $\{0\}$, and the open cone $\hat{\mathcal{V}} \backslash\left\{\nu_{0}\right\}$ over $\mathcal{V}$ is the generic fiber of $\hat{X}$.

\section{$\S 1.4$. Divisorial and quasimonomial valuations}

A divisorial valuation on $X$ is a valuation $\nu$ proportional to the vanishing order $\operatorname{ord}_{E}$ along a prime divisor $E$ in some smooth birational model $Y$ of $X$. If $\nu$ is centered at 0 , then $Y$ can be chosen as $X_{\pi}$ for some $\pi \in \mathfrak{B}$ and $E$ as an exceptional prime (divisor), that is, an irreducible component of $\pi^{-1}(0)$.

A quasi-monomial valuation $\nu$ on $X$ is a valuation that becomes monomial on some smooth birational model $Y$ of $X$. This means the following: there exist a closed point $p$ in $Y$, local coordinates $z=\left(z_{1}, \ldots, z_{n}\right)$ at $p$ and real numbers $w_{1}, \ldots, w_{n} \geq 0$ (not all zero) called weights such that if $f$ in the local ring of $Y$ at $p$ is written as a power series $f=\sum_{\alpha} a_{\alpha} z^{\alpha}$ in multi-index notation, then $\nu$ coincides with the monomial valuation

$$
\nu_{z, w}(f):=\min \left\{w \cdot \alpha, a_{\alpha} \neq 0\right\} .
$$

In terms of the associated absolute value, a valuation is monomial in the $z$ coordinates iff $\left|\sum_{\alpha} a_{\alpha} z^{\alpha}\right|=\max _{\alpha}\left|a_{\alpha} z^{\alpha}\right|$. The center of $\nu$ on $Y$ is then the intersection of all $\left\{z_{i}=0\right\}$ for which $w_{i}>0$. In particular, a divisorial valuation is quasi-monomial (take $w_{i}=0$ for all but one $i$ ) and conversely $\nu$ as above is divisorial iff $w=\left(s_{1}, \ldots, s_{n}\right) \in \mathbf{R}_{+}^{n}$ is proportional to an element of $\mathbf{Q}_{+}^{n}$. The corresponding prime divisor is then the exceptional divisor of a suitable toric blow-up of $Y$ in the coordinates $z_{i}$.

Quasi-monomial valuations can also be characterized as Abhyankar valuations, i.e. real-valued valuations on $X$ for which equality holds in the Abhyankar inequality (1.1), see [ELS, Proposition 4.8].

If $\nu$ is a quasi-monomial valuation on $X$ centered at 0 , then the birational model $Y$ as above can be chosen to be $X_{\pi}$ for some $\pi \in \mathfrak{B}$. More precisely, let $\pi: X_{\pi} \rightarrow X$ be a modification above 0 such that $\pi^{-1}(0)$ is a normal crossings divisor with components $E_{1}, \ldots, E_{N}$, let $Z$ be a component of $E_{I}:=\bigcap_{i \in I} E_{i}$ for some family $I \subset\{1, \ldots, N\}$, and let $w_{i}>0$ be given real numbers for $i \in I$. If $p$ is some point of $Z$, the local equations $z_{i}, i \in I$ of the $E_{i}$ at $p$ can be completed to a local coordinate system $z_{1}, \ldots, z_{n}$ on $X_{\pi}$ at $p$. Then there exists a unique quasi-monomial valuation on $X$ which is monomial on $X_{\pi}$ in the coordinates $z_{1}, \ldots, z_{n}$ with values $\nu\left(z_{i}\right)=w_{i}$ for $i \in I$ and $\nu\left(z_{i}\right)=0$ otherwise. It is easily seen that $\nu$ does not depend on the choice of $p \in Z$ nor on the way 
the $z_{i}, i \in I$ are completed to a coordinate system at $p$. We will refer to $\nu$ as the monomial valuation on $X_{\pi}$ with center $Z$ and value $\nu\left(E_{i}\right)=w_{i}$ on $E_{i}$.

Conversely, it follows from Hironaka's principalization theorem that every quasi-monomial valuation centered at 0 is obtained in that way.

Definition 1.5. We denote by $\hat{\mathcal{V}}_{\text {div }} \subset \hat{\mathcal{V}}\left(\right.$ resp. $\left.\hat{\mathcal{V}}_{\mathrm{qm}} \subset \hat{\mathcal{V}}\right)$ the set of divisorial (resp. quasi-monomial) valuations centered at 0 in $X$, and by $\mathcal{V}_{\text {div }} \subset$ $\mathcal{V}, \mathcal{V}_{\mathrm{qm}} \subset \mathcal{V}$ the corresponding sets of normalized valuations.

Note that every quasi-monomial valuation has home $X$ itself, and in particular the trivial valuation $\nu_{0}$ is definitely not a quasi-monomial valuation. Thus $\hat{\mathcal{V}}_{\text {qm }}$ (resp. $\hat{\mathcal{V}}_{\text {div }}$ ) is just the open cone over $\mathcal{V}_{\text {qm }}$ (resp. $\left.\mathcal{V}_{\text {div }}\right)$.

The vanishing order at $0 \nu_{\mathfrak{m}}(f):=\max \left\{k \in \mathbf{N}^{*}, f \in \mathfrak{m}^{k}\right\}$ is a valuation that plays a special role in $\mathcal{V}$. It is the divisorial valuation associated to the blowup of 0 , and is also the monomial valuation on $X$ (in any local coordinates at 0 ) with all weights equal to 1 . It is normalized, and satisfies the following minimality property: for all $\nu \in \mathcal{V}$ and all $f \in R$ we have $\nu(f) \geq \nu_{\mathfrak{m}}(f)$.

\section{$\S 1.5$. Simple modifications and dual complexes}

We now aim at shedding some light on the geometry of $\mathcal{V}$. Our presentation is strongly inspired by [KS], [KKMS] and [T2].

A modification $\pi \in \mathfrak{B}$ is simple if its exceptional divisor $\pi^{-1}(0)=E_{1}+$ $\cdots+E_{N}$ has simple normal crossings, and is such that $E_{I}:=\bigcap_{i \in I} E_{i}$ is irreducible (or empty) for each $I \subset\{1, \ldots, N\}$. Modifications with simple normal crossings exceptional divisor are cofinite in $\mathfrak{B}$ as a consequence of Hironaka's principalization theorem, and the irreducibility condition can then be reached by further blowing-up components of intersections of exceptional primes sufficiently many times. Thus simple modifications are cofinite in $\mathfrak{B}$.

To a simple modification $\pi$ is attached a simplicial complex which encodes the incidence relations between the $E_{i}$ 's. Its vertices correspond to the $E_{i}$, and its faces to subsets $I \subset\{1, \ldots, N\}$ such that $E_{I}$ is non-empty. We shall now show how to embed the underlying space of this complex into both $\mathbf{R}^{N}$ and into $\mathcal{V}$.

First define $\hat{\Delta}(\pi) \subset \mathbf{R}^{N}$ to be the union of all rational simplicial cones $\hat{\sigma}_{I}:=\sum_{i \in I} \mathbf{R}_{+} e_{i} \subset \mathbf{R}^{I}$, where $I$ runs over all subsets of $\{1, \ldots, N\}$ with $E_{I} \neq \emptyset$, and $\left(e_{1}, \ldots, e_{N}\right)$ is the canonical basis of $\mathbf{R}^{N}$.

Now pick a point $w \in \mathbf{R}^{N}$ lying in $\hat{\Delta}(\pi) \backslash\{0\}$, and let $I=\left\{i, w_{i}>0\right\}$, so that $\hat{\sigma}_{I}$ is the maximal cone to which $w$ belongs. Since $E_{I}$ is non-empty and irreducible, we can consider the quasi-monomial valuation $\nu_{w}$ on $X$ that 
is monomial on $X_{\pi}$ with center $E_{I}$ and value $\nu_{w}\left(E_{i}\right)=w_{i}$ for each $i \in I$, as explained in the last section. One easily verifies

Lemma 1.6. The map $w \mapsto \nu_{w}$ yields an embedding of $\hat{\Delta}(\pi) \backslash\{0\}$ into the set $\hat{\mathcal{V}}_{\mathrm{qm}}$ of quasi-monomial valuations in $\hat{\mathcal{V}}$.

Let us now describe the effect of our choice of normalization. A point $w \in \hat{\Delta}(\pi)$ induces a valuation $\nu_{w}$ belonging to $\mathcal{V}$ iff $\nu_{w}(\mathfrak{m})=\sum_{i} b_{i} w_{i}=1$, with $b_{i}:=\operatorname{ord}_{E_{i}}(\mathfrak{m})$, a positive integer. With this in mind we define $\Delta(\pi)$ as the trace of $\hat{\Delta}(\pi)$ on the integral affine hyperplane $\sum_{i} b_{i} w_{i}=1$. It is a simplicial complex with simplicial faces cut out by integral affine equations, all of the form $\sigma_{I}=\hat{\sigma}_{I} \cap \Delta(\pi)$ for some $I$ with $E_{I}$ non-empty. The above lemma shows that $\Delta(\pi)$ embeds as a topological subspace of $\mathcal{V}$, which will be denoted by $|\Delta(\pi)|$.

We can endow the topological space $|\Delta(\pi)|$ with the structure of integral simplicial complex on $|\Delta(\pi)|$. We refer to [KKMS, pp.69-70] for a general definition. Suffice it to say that $|\Delta(\pi)|$ comes equipped with faces $\left|\sigma_{I}\right|$, defined as the homeomorphic images of the faces $\sigma_{I}$ of $\Delta(\pi)$ by the above embedding into $\mathcal{V}_{\text {qm }}$, and by a free $\mathbf{Z}$-module $\operatorname{Aff}(\pi)_{\mathbf{Z}} \subset C^{0}(|\Delta(\pi)|)$ of rank $N$, induced by the restrictions to $\Delta(\pi)$ of all integral linear functions on $\mathbf{R}^{N}$.

The data $(|\Delta(\pi)|, \operatorname{Aff}(\pi) \mathbf{z})$ enables us to completely recover the simplicial complex $\Delta(\pi) \subset \mathbf{R}^{N}$, up to unimodular transformations. Indeed, an appropriate choice of a $\mathbf{Z}$-basis $f_{1}, \ldots, f_{N}$ for $\operatorname{Aff}(\pi)_{\mathbf{Z}}$ defines a map $|\Delta(\pi)| \rightarrow \mathbf{R}^{N}$ whose image is exactly $\Delta(\pi)$. We thus think of the data $(|\Delta(\pi)|, \operatorname{Aff}(\pi) \mathbf{z})$ as $\Delta(\pi)$ itself, with its faces and its integral affine functions, but embedded in $\mathcal{V}_{\mathrm{qm}}$ instead of an Euclidean space.

Definition 1.7. The dual (simplicial) complex $\Delta(\pi)$ of a simple modification $\pi$ is the integral simplicial complex consisting of the topological space $|\Delta(\pi)| \subset \mathcal{V}_{\text {qm }}$ endowed with the lattice $\operatorname{Aff}(\pi)_{\mathbf{Z}}$ of integral affine functions.

We introduce the following useful terminology.

Definition 1.8. If $\pi$ is a simple modification with exceptional divisors $E_{i}$, and $\mu$ is the blow-up of $X_{\pi}$ with center $E_{I}$ for some $I$, then $\mu$ is called a monomial blow-up. Two simple modifications $\pi$ and $\pi^{\prime}$ are said to be compatible when one is obtained from the other by a sequence of monomial blow-ups.

The subset $|\Delta(\pi)| \subset \mathcal{V}_{\mathrm{qm}}$ is not uniquely determined by $\pi$. If $\pi$ is a simple modification, and $\mu$ is the monomial blow-up of $X_{\pi}$ with center $E_{I}$ for some 
$I$, then $\Delta(\pi \circ \mu)$ is obtained from $\Delta(\pi)$ by a barycentric subdivision of the face $\sigma_{I}$. Hence $|\Delta(\pi \circ \mu)|=|\Delta(\pi)|$ as subsets of $\mathcal{V}_{\mathrm{qm}} \cdot{ }^{3}$ This is essentially the only case: one can show that given two simple modifications $\pi$ and $\pi^{\prime}$, $|\Delta(\pi)|=\left|\Delta\left(\pi^{\prime}\right)\right|$ holds iff there exists another simple modification $\pi^{\prime \prime}$ which is compatible with both $\pi$ and $\pi^{\prime}$. The dual complex $\Delta\left(\pi^{\prime}\right)$ of $\pi^{\prime}$ compatible with $\pi$ is thus obtained by a sequence of barycentric subdivisions of $\Delta(\pi)$. A function on $|\Delta(\pi)|$ will be said to be piecewise integral affine if it belongs to $\operatorname{Aff}\left(\pi^{\prime}\right) \mathbf{z}$ for some $\pi^{\prime}$ compatible with $\pi$.

Definition 1.9. A simplex $|\sigma|$ of $\mathcal{V}_{\mathrm{qm}}$ is a face of $|\Delta(\pi)|$ for a simple modification $\pi$. It is endowed with an integral affine structure $\operatorname{Aff}(|\sigma|)_{\mathbf{z}} \subset$ $C^{0}(|\sigma|)$, defined as the restriction of $\operatorname{Aff}(\pi)_{\mathbf{Z}}$ to $|\sigma|$.

The integral affine structure on a simplex $|\sigma|$ is independent of the choice of $\pi$ above, and functions in $\operatorname{Aff}(|\sigma|) \mathbf{z}$ will be said to be integral affine on $|\sigma|$. If $\pi$ is a simple modification such that $|\sigma|$ is a face of $|\Delta(\pi)|$, then, with notation as above, the elements of $|\sigma|$ can be described as the monomial valuations $\nu_{w}$ on $X_{\pi}$ centered on $E_{I}$ taking values $w_{i}$ on $E_{i}$ for $i \in I$ and such that $\sum_{i \in I} b_{i} w_{i}=1$. The coordinates $t_{i}:=b_{i} w_{i}$ identify $|\sigma|$ with the standard simplex in $\mathbf{R}^{I}$, and $\operatorname{Aff}(|\sigma|) \mathbf{z}$ corresponds to the standard integral functions $t \mapsto \sum_{i} a_{i} t_{i}, a_{i} \in \mathbf{Z}$. The set $\mathcal{V}_{\text {div }} \cap|\sigma|$ of divisorial valuations contained in $|\sigma|$ is exactly the set of points with rational $w$-coordinates. In particular, it is dense in $|\sigma|$.

If $|\sigma|,\left|\sigma^{\prime}\right|$ are two simplices in the same $|\Delta(\pi)|$, then $|\sigma| \cap\left|\sigma^{\prime}\right|$ is either empty or a simplex having integral affine structure $\operatorname{Aff}\left(|\sigma| \cap\left|\sigma^{\prime}\right|\right) \mathbf{z}$ equal to the restriction of both $\operatorname{Aff}(|\sigma|) \mathbf{z}$ and $\operatorname{Aff}\left(\left|\sigma^{\prime}\right|\right) \mathbf{z}$ to $|\sigma| \cap\left|\sigma^{\prime}\right|$.

The main relation between the affine structure on simplices and formal functions in $\hat{R}$ is the following.

Lemma 1.10. If $f \in \hat{R}$ is a formal function, the restriction of $\log |f|$ to any simplex $|\sigma|$ is convex, continuous, and locally piecewise affine.

Proof. Let $\pi$ be a simple modification such that $|\sigma|=\left|\sigma_{I}\right|$ occurs as a face of $|\Delta(\pi)|$ as above. If $p$ is a point of $E_{I}$, and local equations $z_{i}$ of the $E_{i}$ at $p$ have been completed to local coordinates $z_{1}, \ldots, z_{n}$ on $X_{\pi}$ at $p$, we can write $f \circ \pi=\sum_{\alpha} a_{\alpha} z^{\alpha}$. For any $w \in \sigma_{I}$, with corresponding valuation $\nu_{w} \in|\sigma|$, we then have $\nu_{w}(f)=\min \left\{w \cdot \alpha, a_{\alpha} \neq 0\right\}$, so that $\log |f|\left(\nu_{w}\right)=-\nu_{w}(f)$ is the maximum of the family of integral affine functions $w \mapsto-w \cdot \alpha$ such that $a_{\alpha} \neq 0$. The maximum can be taken over finitely many of these functions and then all the conclusions are immediate.

\footnotetext{
${ }^{3}$ Note, however, that $\operatorname{Aff}(\pi) \subsetneq \operatorname{Aff}(\pi \circ \mu)$.
} 


\section{§1.6. Retractions and piecewise affine functions}

Let $\Delta(\pi)$ be the dual complex of a simple modification $\pi$, with exceptional primes $E_{1}, \ldots, E_{N}$. Given any valuation $\nu \in \mathcal{V}$, let $I$ be the family of primes $E_{i}$ that contain the center $Z$ of $\nu$ on $X_{\pi}$, i.e. such that $\nu\left(E_{i}\right)>0$. There exists a unique valuation $r_{\pi}(\nu)$ which is monomial on $X_{\pi}$, with center $E_{I}$ and such that $r_{\pi}(\nu)\left(E_{i}\right)=\nu\left(E_{i}\right)$ for all $i \in I$.

We thus get a continuous retraction map $r_{\pi}: \mathcal{V} \rightarrow|\Delta(\pi)|$, which in fact only depends on the subset $|\Delta(\pi)| \subset \mathcal{V}$. Indeed, it is enough to check that $r_{\pi}=r_{\pi^{\prime}}$ whenever $\pi^{\prime}=\pi \circ \mu$ for a monomial blow-up $\mu$ of $X_{\pi}$, and this is easily done.

Proposition 1.11. For any simple modification $\pi$, there exists a naturally defined retraction map $r_{\pi}: \mathcal{V} \rightarrow|\Delta(\pi)|$. Furthermore, if $\pi^{\prime}$ is any other simple modification dominating $\pi$, the restriction $r_{\pi^{\prime}, \pi}:\left|\Delta\left(\pi^{\prime}\right)\right| \rightarrow|\Delta(\pi)|$ of $r_{\pi}$ to $\left|\Delta\left(\pi^{\prime}\right)\right|$ is integral affine, in the sense that $r_{\pi^{\prime}, \pi}^{*}$ sends $\operatorname{Aff}(\pi)_{\mathbf{Z}}$ into $\operatorname{Aff}\left(\pi^{\prime}\right)_{\mathbf{Z}}$.

Using $r_{\pi}$, we can identify $\operatorname{Aff}(\pi)_{\mathbf{z}}$ with the subgroup $r_{\pi}^{*} \operatorname{Aff}(\pi)_{\mathbf{Z}} \subset C^{0}(\mathcal{V})$.

Definition 1.12. A piecewise affine function on $\mathcal{V}$ is an element of the union $\operatorname{PA}(\mathcal{V})$ of all $\operatorname{Aff}(\pi)_{\mathbf{z}} \otimes \mathbf{R} \subset C^{0}(\mathcal{V})$. It is said to be integral when it belongs to some $\operatorname{Aff}(\pi) \mathbf{z}$.

The inductive system of all simple modifications $\pi$ gives rise to an inductive system of integral simplicial complexes $|\Delta(\pi)|$ with integral affine maps $r_{\pi^{\prime}, \pi}$ : $\left|\Delta\left(\pi^{\prime}\right)\right| \rightarrow|\Delta(\pi)|$. The projective limit $\varliminf_{\pi}|\Delta(\pi)|$ of compact topological spaces is compact, and there is a natural map $\mathcal{V} \rightarrow \varliminf_{\pi}|\Delta(\pi)|$ given by the collection $r_{\pi}$. This map is injective because, for any two distinct valuations $\nu_{1}, \nu_{2} \in \mathcal{V}$ there exists a simple modification $\pi \in \mathfrak{B}$ such that the centers of $\nu_{1}$ and $\nu_{2}$ on $X_{\pi}$ are distinct.

On the other hand, we have seen that any quasi-monomial valuation lies in some $|\Delta(\pi)|$. Whenever $\pi^{\prime}$ dominates $\pi$ the inclusion $|\Delta(\pi)| \subset\left|\Delta\left(\pi^{\prime}\right)\right|$ realizes $\Delta(\pi)$ as a integral simplicial sub-complex of $\Delta\left(\pi^{\prime}\right)$. We thus have injections

$$
\underset{\pi}{\lim _{\longrightarrow}}|\Delta(\pi)|=\mathcal{V}_{\mathrm{qm}} \hookrightarrow \mathcal{V} \hookrightarrow \underbrace{\lim }_{\pi}|\Delta(\pi)| .
$$

The first space being dense in the last, and $\mathcal{V}$ being compact we conclude that $\mathcal{V}=\varliminf^{\lim }|\Delta(\pi)|$. We have thus proved

Theorem 1.13. The space $\mathcal{V}$ with its piecewise integral affine functions $\mathrm{PA}(\mathcal{V})_{\mathbf{Z}}$ can be naturally identified with the projective limit of the system of integral simplicial complexes $r_{\pi^{\prime}, \pi}:\left|\Delta\left(\pi^{\prime}\right)\right| \rightarrow|\Delta(\pi)|$. 
The space $\mathcal{V}_{\mathrm{qm}}$ of quasi-monomial valuations can be naturally identified with the inductive limit $\lim _{\pi}|\Delta(\pi)|$.

In view of the preceding result, it is natural to introduce the strong topology on $\mathcal{V}_{\mathrm{qm}}$ as the inductive limit topology. Since each dual complex is a finite union of simplices, a function $g$ on $\mathcal{V}_{\mathrm{qm}}$ is continuous iff its restriction to every simplex $|\sigma|$ is continuous.

\section{§1.7. Ideals, Cartier divisors and piecewise affine functions}

We now relate piecewise affine functions on $\mathcal{V}$ to primary ideals of $R$ and Cartier divisors on $\mathfrak{X}$.

First note that a Weil divisor $Z \in \operatorname{Div}(\mathfrak{X})$ induces a real-valued function $g_{Z}: \mathcal{V}_{\mathrm{div}} \rightarrow \mathbf{R}$ by setting $g_{Z}(\nu)=\operatorname{ord}_{E}(Z) / \operatorname{ord}_{E}(\mathfrak{m})$ whenever $\nu$ is the normalized valuation proportional to a divisorial valuation $\operatorname{ord}_{E}$. This induces a natural bijection between Weil divisors on $\mathfrak{X}$ and functions on $\mathcal{V}_{\text {div }}$.

Proposition 1.14. If $Z$ is a Cartier divisor on $\mathfrak{X}$, the function $g_{Z}$ : $\mathcal{V}_{\text {div }} \rightarrow \mathbf{R}$ extends by continuity to a function in $\mathrm{PA}(\mathcal{V})$, and this induces an isomorphism $\mathrm{C}-\operatorname{Div}(\mathfrak{X}) \rightarrow \operatorname{PA}(\mathcal{V})$. If $\mathfrak{a}$ is a primary ideal of $R$, the Cartier divisor $Z(\mathfrak{a})$ it determines is associated to the function $\log |\mathfrak{a}|$, and $\operatorname{PA}(\mathcal{V})_{\mathbf{z}}$ coincides with the subgroup of $C^{0}(\mathcal{V})$ generated by $\log |\mathfrak{a}|$ for $\mathfrak{a}$ ranging over all primary ideals of $R$.

Proof. If $\pi$ is a simple modification, the vertices of $\Delta(\pi)$ correspond to the exceptional primes of $\pi$. This yields an identification $\operatorname{Div}(\pi)_{\mathbf{Z}} \rightarrow \operatorname{PA}(\pi)_{\mathbf{z}}$, by sending a divisor $Z_{\pi}$ to the unique function on $\Delta(\pi)$ that is affine on each face and whose value at each vertex $E$ is given by $\operatorname{ord}_{E}\left(Z_{\pi}\right) / \operatorname{ord}_{E}(\mathfrak{m})$. Now, if $\pi^{\prime}=\pi \circ \mu$ is another simple modification, it is easily checked that $\mu^{*}: \operatorname{Div}(\pi) \rightarrow$ $\operatorname{Div}\left(\pi^{\prime}\right)$ corresponds to the map $\left(r_{\pi, \pi^{\prime}}\right)^{*}: \operatorname{PA}(\pi) \rightarrow \operatorname{PA}\left(\pi^{\prime}\right)$. This proves that the above isomorphisms induce an isomorphism between $\mathrm{C}-\operatorname{Div}(\mathfrak{X})_{\mathbf{Z}}=$ $\lim _{\longrightarrow} \operatorname{Div}(\pi)_{\mathbf{z}}$ and $\operatorname{PA}(\mathcal{V})_{\mathbf{z}}=\lim _{\longrightarrow} \operatorname{PA}(\pi)_{\mathbf{z}}$.

Now a given $\mathbf{Z}$-divisor $\vec{D}$ on some $X_{\pi}$ can be written as a difference of two $\mathbf{Z}$-divisors $D_{1}-D_{2}$, where each $D_{i}$ is generated by its global sections on $X_{\pi}$. This means that $\mathcal{O}\left(D_{i}\right)=\pi^{-1} \mathfrak{a}_{i}$ for some primary ideals $\mathfrak{a}_{i}$ in $R$, and this shows that the group generated by functions of the form $\log |\mathfrak{a}|$ with $\mathfrak{a}$ a primary ideal coincides with $\operatorname{PA}(\mathcal{V})_{\mathbf{z}}$.

Proposition 1.15. The subspace $\operatorname{PA}(\mathcal{V})$ is dense in $C^{0}(\mathcal{V})$, endowed with the topology of uniform convergence. 
Proof. This is a straightforward consequence of the so-called lattice version of the Stone-Weierstrass theorem. Indeed, we have $|\mathfrak{a}+\mathfrak{b}|=\max (|\mathfrak{a}|,|\mathfrak{b}|)$ as functions on $\mathcal{V}$ for any two primary ideals $\mathfrak{a}, \mathfrak{b}$, and it follows immediately that the closure of the $\mathbf{Q}$-vector subspace of $C^{0}(\mathcal{V})$ spanned by all functions of the form $\log |\mathfrak{a}|$ is a closed $\mathbf{R}$-subspace of $C^{0}(\mathcal{V})$ stable by taking max. Since it also separates points of $\mathcal{V}$ in a tautological way, the lattice version of the Stone-Weierstrass theorem implies that this space is equal to $C^{0}(\mathcal{V})$. But it also coincides with the closure of $\mathrm{PA}(\mathcal{V})$, and the result follows.

\section{$\S 1.8 . \quad$ Contractibility}

We conclude with the following result due to Berkovich [Be, p.116]. It is also a special case of a more general result of [T2].

Theorem $1.16([\mathrm{Be}],[\mathrm{T} 2]) . \quad$ The spaces $\mathcal{V}, \mathcal{V}_{\mathrm{qm}}$ are contractible.

Proof. Let $x_{1}, \ldots, x_{n}$ be coordinates on $X$ at 0 , and denote by $\mathcal{V}_{\mathrm{m}} \subset \mathcal{V}$ the space of all monomial valuations, i.e. determined by their values on $x_{i}$. Clearly $\mathcal{V}_{\mathrm{m}}$ can be identified with $\left\{\left(s_{i}\right) \in[1,+\infty]^{n}\right.$, $\left.\min s_{i}=+1\right\}$, and is thus contractible. Now define the retraction $r: \mathcal{V} \rightarrow \mathcal{V}_{\mathrm{m}}$ by:

$$
r(\nu)(f)=\min _{\alpha}\left\{\nu\left(a_{\alpha} x^{\alpha}\right)\right\}=\min \left\{\nu\left(x^{\alpha}\right), a_{\alpha} \neq 0\right\},
$$

where $f=\sum_{\alpha} a_{\alpha} x^{\alpha}$ in multi-index notation. We construct a homotopy $h_{t}$ from $r$ to the identity as follows. For each multi-index $\beta \in \mathbf{N}^{n}$, let $T_{\beta}$ be the truncation operator on $k\left[x_{1}, \ldots, x_{n}\right]$ given by $T_{\beta}\left(\sum_{\alpha} a_{\alpha} x^{\alpha}\right)=\sum_{\alpha \geq \beta} a_{\alpha} x^{\alpha}$. Here $\alpha=\left(\alpha_{i}\right) \geq\left(\beta_{i}\right)$ iff $\alpha_{i} \geq \beta_{i}$ for each $i$. Then for $t \in[0,1]$, we set

$$
h_{t}(\nu)(f):=\min _{\beta}\left\{\nu\left(T_{\beta}(f)\right)-|\beta| \log t\right\} .
$$

Note that the minimum is attained for only finitely many $\beta$ 's. To see that the function $h_{t}(\nu)$ is a valuation, it is enough to check its additivity on the product of two functions. Pick $f, g \in R$, and let $\alpha$ (resp. $\beta$ ) be the minimal index for the lexicographic order on $\mathbf{N}^{n}$ such that $h_{t}(\nu)(f)=\nu\left(T_{\alpha}(f)\right)-|\alpha| \log t$ $\left(\right.$ resp. $\left.h_{t}(\nu)(g)=\nu\left(T_{\beta}(g)\right)-|\beta| \log t\right)$. Set $\gamma=\alpha+\beta$. Then $\nu\left(T_{\gamma}(f g)\right)=$ $\nu\left(\sum_{\alpha^{\prime}+\beta^{\prime}=\gamma} T_{\alpha^{\prime}}(f) T_{\beta^{\prime}}(g)\right)=\nu\left(T_{\alpha}(f)\right)+\nu\left(T_{\beta}(g)\right)$, hence $h_{t}(\nu)(f g) \leq h_{t}(\nu)(f)+$ $h_{t}(\nu)(g)$. The reverse inequality is clear.

It is easy to see that $h_{0}$ is the identity on $\mathcal{V}$, and $h_{1}$ coincides with $r$ in view of the relation $\min _{\beta} \nu\left(\sum_{\alpha \geq \beta} a_{\alpha} x^{\alpha}\right)=\min _{\beta} \nu\left(a_{\beta} x^{\beta}\right)$. Continuity of $h_{t}(\nu)$ in $(t, \nu)$ is also easily checked, and $h_{t}$ is the identity on $\mathcal{V}_{\mathrm{m}}$ for each $t$. 
For each simple modification $\pi$, the retraction $r_{\pi}: \mathcal{V} \rightarrow|\Delta(\pi)|$ can also be deformed to the identity map of $\mathcal{V}$ relatively to $|\Delta(\pi)|$. This is done in [T2] using the language of Berkovich spaces, relying on the fact that the normal crossing exceptional divisor of $\pi$ endows $X_{\pi}$ with a toroidal structure.

Remark 1.17. The valuation spaces $\mathcal{V}_{\mathrm{qm}}$ and $\mathcal{V}$ in dimension $n=2$ were studied by the last two authors in [FJ1]. In this case, $\mathcal{V}$ is naturally a tree-the valuative tree - and the piecewise affine integral structure corresponds to the parameterization of $\mathcal{V}$ by skewness. Moreover, $\mathcal{V}_{\mathrm{qm}}$ is a dense subtree of $\mathcal{V}$ obtained by removing all the ends of $\mathcal{V}$. This makes it possible to understand the valuations in $\mathcal{V} \backslash \mathcal{V}_{\mathrm{qm}}$. In higher dimensions, the structure of $\mathcal{V} \backslash \mathcal{V}_{\mathrm{qm}}$ is much more complicated. For instance, it contains the valuation space $\mathcal{V}_{Y}$ for each smooth (formal) subvariety $Y \subset X$ through 0 . In dimension two, $Y$ is a germ of a curve, and $\mathcal{V}_{Y}$ is then a singleton, called a curve valuation.

\section{$\S 2 . \quad$ Positivity and Convexity}

\section{$\S 2.1 . \quad$ Nef Weil divisors}

Recall that to any ideal $\mathfrak{a} \subset \hat{R}$ is associated a Weil divisor $Z(\mathfrak{a}) \leq 0$ which is Cartier iff $\mathfrak{a}$ is primary. For any two ideals $\mathfrak{a}$ and $\mathfrak{b}$ in $\hat{R}$, we have

$$
\begin{aligned}
Z(\mathfrak{a} \cdot \mathfrak{b}) & =Z(\mathfrak{a})+Z(\mathfrak{b}) ; \\
Z(\mathfrak{a}+\mathfrak{b}) & =\max \{Z(\mathfrak{a}), Z(\mathfrak{b})\} .
\end{aligned}
$$

Here the max is taken component-wise. This implies that the set of all $Z(\mathfrak{a})$ 's is a semi-group preserved by taking max. It is the smallest such semi-group containing the divisors $Z(f)$ determined by all $f \in \hat{R}$, since $Z(\mathfrak{a})=\max _{k} Z\left(f_{k}\right)$ whenever the $f_{k}$ 's generate $\mathfrak{a}$.

Definition 2.1. The cone of nef Weil divisors in $\operatorname{Div}(\mathfrak{X})$ is defined as the closed convex cone in $\operatorname{Div}(\mathfrak{X})$ generated by the divisors $Z(\mathfrak{a})$, where $\mathfrak{a}$ runs over all ideals of $\hat{R}$.

Note that a nef Weil divisor $Z$ satisfies $Z \leq 0$, by continuity. If $\mathfrak{a}$ is an arbitrary ideal, then we have $Z(\mathfrak{a})=\lim _{k \rightarrow \infty} Z\left(\mathfrak{a}+\mathfrak{m}^{k}\right)$, so that the $Z(\mathfrak{a})$ 's with $\mathfrak{a}$ a primary ideal of $R$ already generate the nef cone. Since the $Z(\mathfrak{a})$ 's make up a semi-group preserved by taking max, the nef cone of $\operatorname{Div}(\mathfrak{X})$ is also preserved by the same operation.

In the special case of Cartier divisors, we have the following characterization, which explains the terminology. 
Proposition 2.2. $\quad$ A Cartier divisor $Z$ on $\mathfrak{X}$ is nef (as a Weil divisor) iff $Z_{\pi}$ is $\pi$-nef on $X_{\pi}$ for some (hence any) determination $\pi$ of $Z$, that is, $Z_{\pi}$ has non-negative degree on any compact curve contained in $\pi^{-1}(0)$.

Lemma 2.3. Let $Z$ be a nef Weil divisor on $\mathfrak{X}$, and let $Z_{\pi}$ be its incarnation on some $X_{\pi}$. For any exceptional prime $E$ of $\pi$, the restriction $Z_{\pi} \mid E$ of the numerical class of $Z_{\pi}$ to $E$ is pseudo-effective, i.e. it is a limit of classes of effective $\mathbf{Q}$-divisors on $E$.

Proof. Since the set of pseudo-effective classes of $E$ is a closed convex cone, it is enough to check this when $Z=Z(\mathfrak{a})$ for some primary ideal $\mathfrak{a}$. But $Z_{\pi}$ is the divisorial part of $\pi^{-1} \mathfrak{a}$, thus the base-locus of the global sections of $\mathcal{O}\left(Z_{\pi}\right)$ on $X_{\pi}$ has codimension at least 2 . It follows that $E$ cannot be contained in this base-locus, so $\mathcal{O}_{E}\left(Z_{\pi}\right)$ is a line bundle with at least one non-zero global section. In particular, it is pseudo-effective.

Proof of Proposition 2.2. Let $\pi$ be a determination of $Z$. If $Z_{\pi}$ is not $\pi$-nef on $X_{\pi}$, there exists a curve $C$ in $\pi^{-1}(0)$ such that the numerical class $Z_{\pi} \mid C$ is not pseudo-effective. Now pick $\pi^{\prime}=\pi \circ \mu \in \mathfrak{B}$ dominating the blow-up of $C$ in $X_{\pi}$, and let $E$ be an exceptional prime of $\pi^{\prime}$ dominating $C$. Then the restriction of $\mu^{*} Z_{\pi}=Z_{\pi^{\prime}}$ to $E$, which is the same as $(\mu \mid E)^{*}\left(Z_{\pi} \mid C\right)$, cannot be pseudo-effective since $Z_{\pi} \mid C$ is not. This leads to a contradiction in view of the lemma, since $Z_{\pi^{\prime}}$ is the incarnation of the nef divisor $Z$ on $X_{\pi^{\prime}}$.

We record the following monotonicity property of nef divisors.

Proposition 2.4. Let $Z$ be a nef Weil divisor on $\mathfrak{X}$, and let $Z_{\pi} \in$ $\operatorname{Div}(\mathfrak{X})$ be the incarnation of $Z$ on $X_{\pi}$, viewed as a Cartier divisor on $\mathfrak{X}$. Then $Z \leq Z_{\pi}$.

In particular, any nef Weil divisor $Z \neq 0$ satisfies $Z \leq c Z(\mathfrak{m})$ for some $c>0$.

Proof. Since $Z \mapsto Z_{\pi}$ is linear and continuous we can assume $Z=Z(\mathfrak{a})$ for some primary ideal $\mathfrak{a}$. Let $\pi^{\prime}=\pi \circ \mu$ be a determination of $Z$ and write $L:=Z_{\pi^{\prime}}$. Then $\mathcal{O}(L)$ is generated by its global sections with respect to $\pi^{\prime}$, thus a fortiori with respect to $\mu$. In other words, we have $\mathcal{O}(L)=\mu^{*} \mu_{*} \mathcal{O}(L)$. Now the sheaf of ideals $\mathcal{O}\left(\mu_{*} L\right)$ contains $\mu_{*} \mathcal{O}(L)$ since $L \leq 0$, so that $\mathcal{O}(L) \subset \mathcal{O}\left(\mu^{*} \mu_{*} L\right)$. Since $L$ is exceptional, this implies $Z=Z_{\pi^{\prime}} \leq Z_{\pi}$.

The proof also shows that if $Z$ is any nef Weil divisor on $\mathfrak{X}$, then $Z_{\pi^{\prime}} \leq Z_{\pi}$ whenever $\pi^{\prime} \geq \pi$, and $Z=\inf _{\pi} Z_{\pi}$. 


\section{$\S 2.2$. Formal psh functions}

We now interpret the previous positivity notion at the level of functions on the valuation space $\mathcal{V}$. The main technical tool required to study themmultiplier ideals - will be introduced in the next section.

Definition 2.5. A function $g: \mathcal{V}_{\mathrm{qm}} \rightarrow \mathbf{R}$ is called a formal psh function iff it is continuous in the strong topology and if the Weil divisor $Z(g)$ on $\mathfrak{X}$ determined by $g \mid \mathcal{V}_{\text {div }}$ is nef.

As should be expected from the name, formal psh functions share many properties with the usual psh functions on $\mathbf{C}^{n}$. In particular, $\log |f|$ is a formal psh function for any formal function $f \in \hat{R}$, see Lemma 1.10.

Remark 2.6. We will prove later on that any nef Weil divisor $W$ on $\mathfrak{X}$ can conversely be written (in a unique way) as $W=Z(g)$ for some formal psh function $g$ (see Proposition 3.11), so that nef Weil divisors on $\mathfrak{X}$ and formal psh functions on $\mathcal{V}$ are two manifestations of the same object. Let us already here point out what the difficulty is. Given a simplex in $|\sigma|$ in $\mathcal{V}$ we must show that the function $g:|\sigma| \cap \mathcal{V}_{\text {div }} \rightarrow \mathbf{R}$ defined by $W$ extends to a continuous, convex function on $|\sigma|$. Suppose $|\sigma|$ is one-dimensional, for simplicity, so that $|\sigma|$ can be identified with the interval $[0,1]$ and $|\sigma| \cap \mathcal{V}_{\text {div }}$ with $[0,1 \mid \cap \mathbf{Q}$. The nefness assumption on $W$ implies that $g:[0,1] \cap \mathbf{Q} \rightarrow \mathbf{R}$ is convex. However, while $\mathrm{g}$ then automatically extends as a continuous function on the open interval $(0,1)$, there may not be a continuous extension to the closed interval $[0,1]$. An example is given by $g(x)=-x$ for $0 \leq x<1$ and $g(1)=0$.

If $Z$ is a Weil divisor on $\mathfrak{X}$ and $\pi$ is a simple modification, recall that we denoted by $Z_{\pi}$ the Cartier divisor in $\mathfrak{X}$ determined by the incarnation of $Z$ in $X_{\pi}$. In terms of the associated function $g_{Z}$ on $\mathcal{V}_{\text {div }}$, we see that $Z_{\pi}$ corresponds to the unique affine function in $\operatorname{Aff}(\pi)$ that coincides with $g_{Z}$ on the vertices of $\Delta(\pi)$, i.e. to the piecewise affine interpolation of $g_{Z}$ on $\Delta(\pi)$. Now suppose that $g$ is a formal psh function. Then by Proposition 2.4, $Z(g) \leq Z(g)_{\pi}$ for any $\pi$. Both functions $g_{Z_{\pi}}$ and $g \circ r_{\pi}$ belong to $r_{\pi}^{*} C^{0}(|\Delta(\pi)|)$, but they do not coincide, since the restriction of $g$ to $|\Delta(\pi)|$ is not piecewise affine in general. Using the notion of compatible blowups from Definition 1.8, we have:

$$
g \circ r_{\pi}=\inf \left\{g_{Z_{\pi^{\prime}}}, \pi^{\prime} \text { compatible with } \pi\right\} .
$$

Using this fact, we conclude 
Proposition 2.7. $\quad$ For any dual complex $\Delta(\pi)$ and any formal psh function $g$, we have $g \leq g \circ r_{\pi}$.

In dimension $2, Z_{\pi}$ is nef whenever $Z$ is, and thus $g \circ r_{\pi}$ is formal psh when $g$ is. These two properties fail in the higher dimensional case.

If $\pi^{\prime} \geq \pi$ are two simple modifications, we have $|\Delta(\pi)| \subset\left|\Delta\left(\pi^{\prime}\right)\right|$, thus $r_{\pi} \circ r_{\pi^{\prime}}=r_{\pi}$. Hence $g \circ r_{\pi}$ decreases as $\pi$ increases. Using this, we extend $g$ to $\mathcal{V} \backslash \mathcal{V}_{\mathrm{qm}}$ as follows:

Definition 2.8. Given a formal psh function $g: \mathcal{V}_{\mathrm{qm}} \rightarrow \mathbf{R}$, we extend $g$ to $\mathcal{V}$ by setting

$$
g(\nu):=\inf _{\pi} g \circ r_{\pi}(\nu)
$$

for any valuation $\nu \in \mathcal{V}$.

Since the restriction of $g$ to any dual complex $|\Delta(\pi)|$ is continuous, we see that $g \circ r_{\pi}$ is continuous in the weak topology of $\mathcal{V}$ for each $\pi$, and thus the extension $g: \mathcal{V} \rightarrow[-\infty, 0]$ is upper semicontinuous, as in the case of the usual psh functions.

\section{$\S 3 . \quad$ Valuative Multiplier Ideals}

The goal of this section is to attach to each nef Weil divisor $W$ on $\mathfrak{X}$ a valuative multiplier ideal $\mathcal{L}^{2}(W) \subset \hat{R}$, in such a way that $\mathcal{L}^{2}(c Z(\mathfrak{a}))$ coincides with the usual multiplier ideal $\mathcal{J}\left(\mathfrak{a}^{c}\right)$ when $\mathfrak{a}$ is a primary ideal of $R$ and $c>0$. We will then show that the nef Weil divisors $\frac{1}{k} Z\left(\mathcal{L}^{2}(k W)\right)$ approximate in a very precise way a given nef Weil divisor $W$ from above. This will enable us to further investigate formal psh functions. In particular, it will turn out that formal psh functions and nef Weil divisors are equivalent notions.

\section{§3.1. Thinness}

Consider a modification $\pi: X_{\pi} \rightarrow X$ in $\mathfrak{B}$. Denote by $K_{\pi}$ the relative canonical divisor of $\pi$. It is the effective divisor on $X_{\pi}$ determined by the Jacobian determinant $J \pi$ of $\pi$. When $\pi^{\prime} \geq \pi, \pi^{\prime}=\pi \circ \mu$, the chain rule yields $K_{\pi^{\prime}}=\mu^{*} K_{\pi}+K_{\mu}$, where $K_{\mu}$, the relative canonical divisor of $\mu$, is $\mu$-exceptional. Thus there exists a unique Weil divisor $K$ on $\mathfrak{X}$, the canonical divisor, whose incarnation on each $X_{\pi}$ is $K_{\pi}$. For our purposes, a different but closely related Weil divisor will be important.

Definition 3.1. The thin divisor $A$ is the Weil divisor on $\mathfrak{X}$ whose incarnation in $X_{\pi}$ equals $A_{\pi}:=K_{\pi}+\sum E$ where $E$ ranges over all exceptional primes of $\pi$. The corresponding function $A: \mathcal{V}_{\mathrm{div}} \rightarrow \mathbf{R}_{+}$is called thinness. 
Let $|\sigma|$ be a simplex in $\mathcal{V}_{\mathrm{qm}}$, determined by a collection $E_{i}, i \in I$ of exceptional primes of a simple modification $\pi$. If $\nu \in|\sigma|$ is divisorial, a standard computation (see for instance [Ho]) shows that $A(\nu)=\sum_{i \in I} w_{i} A\left(\operatorname{ord}_{E_{i}}\right)$ with $w_{i}=\nu\left(E_{i}\right)$. This yields:

Proposition 3.2. The thinness function $A: \mathcal{V}_{\mathrm{div}} \rightarrow \mathbf{R}_{+}$extends uniquely to a strongly continuous function $A: \mathcal{V}_{\mathrm{qm}} \rightarrow \mathbf{R}_{+}$, which is integral affine on each simplex.

The thinness function satisfies the following monotonicity property.

Proposition 3.3. For any simple modification $\pi$, we have $A \geq A \circ r_{\pi}$ on $\mathcal{V}_{\mathrm{qm}}$, and the inequality is strict outside the dual complex $|\Delta(\pi)|$.

The proof is a standard computation, cf. [Ko, Lemma 3.11].

In particular we have $A \geq A\left(\nu_{\mathfrak{m}}\right)=\operatorname{dim} X$ on $\mathcal{V}_{\mathrm{qm}}$. We also see that $A \circ r_{\pi}: \mathcal{V}_{\mathrm{qm}} \rightarrow \mathbf{R}$ is increasing with respect to $\pi$, so we can extend $A$ to all of $\mathcal{V}$ as follows.

Definition 3.4. The thinness function $A: \mathcal{V} \rightarrow[0,+\infty]$ is the lower semicontinuous function defined by $A=\sup _{\pi} A \circ r_{\pi}$.

Beware that the Weil divisor $-A$ on $\mathfrak{X}$ is not nef, i.e. $-A$ is not a formal psh function on $\mathcal{V}$.

\section{§3.2. Multiplier ideals of ideals}

We start by recalling the usual geometric definition of the multiplier ideals following [L, §9.2].

Let $c>0$ and let $\mathfrak{a} \subset R$ be an ideal (not necessarily primary). Pick a $\log$-resolution $\pi: X^{\prime} \rightarrow X$ of $\mathfrak{a}$. Then $f \in R$ lies in the multiplier ideal $\mathcal{J}\left(\mathfrak{a}^{c}\right)$ iff $\operatorname{ord}_{E}(f \circ \pi)+\operatorname{ord}_{E}\left(K_{\pi}\right) \geq\left[c \operatorname{ord}_{E}\left(\pi^{-1} \mathfrak{a}\right)\right]$ for ${ }^{4}$ any prime divisor $E$ of $X^{\prime}$. If $\mathfrak{a}$ is primary, then $\pi$ can be taken to lie in $\mathfrak{B}$, and the above condition only needs to be tested for the $\pi$-exceptional primes $E$. Using thinness, it can then be rewritten as $\operatorname{ord}_{E}(f)+A\left(\operatorname{ord}_{E}\right)>c \operatorname{ord}_{E}(\mathfrak{a})$.

The multiplier ideal $\mathcal{J}\left(\mathfrak{a}^{c}\right)$ satisfies the following fundamental subadditivity property, first established in [DEL]. We refer to $[\mathrm{L}, \S 9.5]$ for a proof.

Theorem 3.5. Let $\mathfrak{a}$ and $\mathfrak{b}$ be ideals in $R$. Then $\mathcal{J}\left(\mathfrak{a}^{c} \cdot \mathfrak{b}^{d}\right) \subset \mathcal{J}\left(\mathfrak{a}^{c}\right)$. $\mathcal{J}\left(\mathfrak{b}^{d}\right)$ for any $c, d>0$.

\footnotetext{
${ }^{4}$ Here $[x]$ is the integral part (rounded down) of the real-number $x$.
} 


\section{§3.3. Multiplier ideals of nef Weil divisors}

Definition 3.6. $\quad$ Let $W$ be a nef Weil divisor on $\mathfrak{X}$. The multiplier ideal $\mathcal{L}^{2}(W)$ is defined as the stationary limit as $\varepsilon>0$ decreases to 0 of the ideals

$$
\{f \in \hat{R}, Z(f) \leq(1+\varepsilon) W+A\}
$$

If $g$ is a formal psh function on $\mathcal{V}$, then we define $\mathcal{L}^{2}(g):=\mathcal{L}^{2}(Z(g))$.

Note that we consider arbitrary formal functions $f \in \hat{R}$, not just in $R$. Since $Z \leq 0$, the ideals in (3.1) form an increasing sequence as $\varepsilon>0$ decreases, hence are indeed stationary by the Noetherian property. Since each of these ideals is integrally closed, so is the multiplier ideal $\mathcal{L}^{2}(W)$. Note however that it is not primary in general. The definition is arranged so that $\mathcal{L}^{2}(W)=$ $\mathcal{L}^{2}((1+\varepsilon) W)$ for every $\varepsilon>0$ small enough. Also, it coincides with the usual multiplier ideal when $W=c Z(\mathfrak{a})$ for some ideal $\mathfrak{a}$ of $R$ :

Proposition 3.7. When $\mathfrak{a} \subset R$ is an ideal and $c>0$, the ideal $\mathcal{L}^{2}(c Z(\mathfrak{a}))$ $\cap R$ coincides with the usual multiplier ideal $\mathcal{J}\left(\mathfrak{a}^{c}\right)$.

Remark 3.8. Over the complex numbers, if $f_{1}, \ldots, f_{k}$ are generators of $\mathfrak{a}$, then $f$ is in the multiplier ideal of $\mathfrak{a}^{c}$ iff $|f| / \max _{j}\left|f_{j}\right|^{c}$ is locally squareintegrable at 0 . This explains our notation $\mathcal{L}^{2}$ above.

Proof of Proposition 3.7. This is essentially trivial when $\mathfrak{a}$ is primary, but the non-primary case requires some care since $\mathcal{L}^{2}(c \log |\mathfrak{a}|)$ is defined by imposing conditions at valuations centered at 0 on $X$ only.

Set $Z:=c Z(\mathfrak{a})$ and $W=Z\left(\mathcal{J}\left(\mathfrak{a}^{c}\right)\right)$. We know that $\operatorname{ord}_{E}(W)<\operatorname{ord}_{E}(Z)+$ $A\left(\operatorname{ord}_{E}\right)$ for any divisorial valuation $\operatorname{ord}_{E}$ on $X$ (not necessarily centered at $0)$. Indeed, $E$ can be realized as an exceptional prime of a log-resolution of $\mathfrak{a}$. As a consequence, $W<Z+A$ as Weil divisors on $\mathfrak{X}$. Now, it is well known that $\mathcal{J}\left(\mathfrak{a}^{c}\right)=\mathcal{J}\left(\mathfrak{a}^{(1+\varepsilon) c}\right)$ for $0<\varepsilon \ll 1$, so we get $W<(1+\varepsilon) Z+A$. Thus $\mathcal{J}\left(\mathfrak{a}^{c}\right) \subset \mathcal{L}^{2}(Z) \cap R$.

To get the reverse inclusion, let $f \in \mathcal{L}^{2}(Z) \cap R$, and pick a log-resolution $\pi: X^{\prime} \rightarrow X$ of $\mathfrak{a}$. Let $\sum_{i} r_{i} E_{i}$ be the effective divisor defined by $\pi^{-1} \mathfrak{a}$. By definition, $f$ belongs to $\mathcal{J}\left(\mathfrak{a}^{c}\right)$ iff $\operatorname{ord}_{E_{i}}(f)>c r_{i}-a_{i}$ for each prime $E_{i}$ of $\pi$, with $a_{i}:=1+\operatorname{ord}_{E_{i}}(J \pi)$. (Note that $\pi$ may not contract $E_{i}$ onto 0 .)

Let $E=E_{i}$ be one of these primes, pick a point $p$ of $E \cap \pi^{-1}(0)$ and let $z_{1}$ be a local equation of $E$ at $p$, completed to a local system of coordinates $z_{1}, \ldots, z_{n}$ on $X^{\prime}$ at $p$. If we let $\nu_{w}$ be the monomial valuation on $X^{\prime}$ in the coordinates $z_{1}, \ldots, z_{n}$ with weights $\nu\left(z_{i}\right)=w_{i}$, then $\operatorname{ord}_{E}=\nu_{w}$ for $w=(1,0, \ldots, 0)$, and 
the center of $\nu_{w}$ on $X$ is $\pi(p)=0$ if $w_{i}>0$ for all $i$. Since $f \in \mathcal{L}^{2}(Z)$, we deduce that $\nu_{w}(f) \geq(1+\varepsilon) c \nu_{w}(\mathfrak{a})-A\left(\nu_{w}\right)$ for each $w$ with all $w_{i}>0$. But clearly $\nu_{w}(\mathfrak{a}) \rightarrow \operatorname{ord}_{E}(\mathfrak{a})$ and $\nu_{w}(f) \rightarrow \operatorname{ord}_{E}(f)$ as $w>0$ converges to $(1,0, \ldots, 0)$, and $A\left(\nu_{w}\right)=w_{1}+\cdots+w_{n}+\nu_{w}(J \pi)$ converges to $1+\operatorname{ord}_{E}(J \pi)$. We deduce that $\operatorname{ord}_{E}(f) \geq(1+\varepsilon) c \operatorname{ord}_{E}(\mathfrak{a})-\left(1+\operatorname{ord}_{E}(J \pi)\right)$ for each $E$, and in particular $f \in \mathcal{J}\left(\mathfrak{a}^{c}\right)$ as was to be shown.

We will prove in Section 3.6 that the valuative multiplier ideals satisfy the following fundamental properties, extending those of the usual multiplier ideals.

Theorem 3.9. If $W$ is a nef Weil divisor on $\mathfrak{X}$, then

$$
\left.W \leq Z\left(\mathcal{L}^{2}(W)\right)\right) \leq W+A .
$$

Theorem 3.10. If $W_{1}$ and $W_{2}$ are two nef Weil divisors on $\mathfrak{X}$, then

$$
\mathcal{L}^{2}\left(W_{1}+W_{2}\right) \subset \mathcal{L}^{2}\left(W_{1}\right) \cdot \mathcal{L}^{2}\left(W_{2}\right) .
$$

in $\hat{R}$.

\section{§3.4. Consequences for formal psh functions}

Using Theorems 3.9 and 3.10 we shall establish two important facts. The first is that nef Weil divisors and formal psh functions are equivalent notions, as already mentioned above.

Proposition 3.11. For any nef Weil divisor $W$, there exists a unique formal psh function $g: \mathcal{V} \rightarrow[-\infty, 0]$ such that $W=Z(g)$. Moreover, $g$ is continuous and convex on any simplex in $\mathcal{V}$.

Proof. Let $g: \mathcal{V}_{\text {div }} \rightarrow \mathbf{R}$ be the function associated to $W$. We have to show that $g$ can be continuously extended to $\mathcal{V}_{\mathrm{qm}}$ endowed with its strong topology. Concretely, this means that $g$ extends to a continuous function on each simplex $|\sigma|$ in $\mathcal{V}$.

The apparent difficulty is that $|\sigma|$ is a closed simplex. Indeed, the nefness of $W$ can be shown to imply that $g$ extends uniquely to a convex (and hence continuous!) function on the interior of $|\sigma|$. However, the behavior of $g$ at the boundary of $|\sigma|$ is harder to control, and that is why we use multiplier ideals.

For each $k>0$, set $g_{k}:=\frac{1}{k} \log \left|\mathcal{L}^{2}(k W)\right|$. This is a formal psh function, and $\left|g-g_{k}\right| \leq A / k$ on $\mathcal{V}_{\text {div }}$ by Theorem 3.9. Since $A$ is bounded on $|\sigma|$, 
$\left(g_{k}\right)_{1}^{\infty}$ is a Cauchy sequence on $|\sigma|$, hence converges uniformly to a continuous function. It is clear that the function thus obtained is the extension of $g$ that we were looking for. The convexity of $g$ on simplices in $\mathcal{V}$ is a consequence of Proposition 2.7.

Corollary 3.12. If $g: \mathcal{V}_{\mathrm{qm}} \rightarrow \mathbf{R}$ is a formal psh function on $\mathcal{V}$, then $f \in \mathcal{L}^{2}(g)$ iff there exists $\varepsilon>0$ such that $\log |f| \leq(1+\varepsilon) g+A$ on $\mathcal{V}_{\mathrm{qm}}$.

Proof. This follows from the definition of $\mathcal{L}^{2}(g)=\mathcal{L}^{2}(Z(g))$ and the strong continuity of $g, A$ and $\log |f|$ on $\mathcal{V}_{\mathrm{qm}}$.

As a second consequence of Theorem 3.9 and 3.10 we obtain the following approximation result.

Proposition 3.13. For any formal psh function $g$ on $\mathcal{V}$, there exists a countable sequence $\left(\mathfrak{a}_{k}\right)_{1}^{\infty}$ of primary ideals and real numbers $c_{k}>0$ such that $g_{k}:=c_{k} \log \left|\mathfrak{a}_{k}\right|$ decreases to $g$ on all of $\mathcal{V}$ as $k \rightarrow \infty$.

Proof. Set $\mathfrak{a}_{k}=\mathcal{L}^{2}\left(2^{k} g\right)+\mathfrak{m}^{4^{k}}$ and $c_{k}=2^{-k}$. By Theorems 3.9 and 3.10, the sequence $h_{k}:=2^{-k} \log \left|\mathcal{L}^{2}\left(2^{k} g\right)\right|$ decreases to $g$, hence $g_{k}=\max \left(h_{k},-2^{k}\right)$ also decreases to $g$ on $\mathcal{V}_{\mathrm{qm}}$. We now show that this property automatically extends to $\mathcal{V} \backslash \mathcal{V}_{\mathrm{qm}}$. Let $\nu$ belong to the latter set, and pick $t>g(\nu)$ (which might be $-\infty)$. By the definition of $g(\nu)$, there exists $\pi$ large enough so that $g\left(r_{\pi}(\nu)\right)<t$, and then $g_{k}\left(r_{\pi}(\nu)\right)<t$ for $k$ large enough. But we also have $g(\nu) \leq g_{k}(\nu) \leq g_{k}\left(r_{\pi}(\nu)\right)$ since $g_{k}$ is formal psh, and it follows that $g_{k}(\nu)$ decreases to $g(\nu)$.

We would like to stress the analogy with the usual complex case, where each psh function can be written as the decreasing limit of smooth psh functions.

Finally we show that formal psh functions satisfy the following uniform Izumi-type bound.

Proposition 3.14. For every $\nu \in \mathcal{V}_{\mathrm{qm}}$, there exists $C=C(\nu)>0$ such that

$$
C g\left(\nu_{\mathfrak{m}}\right) \leq g(\nu) \leq g\left(\nu_{\mathfrak{m}}\right)
$$

for each formal psh function $g$. In particular, either $\max _{\mathcal{V}} g<0$ or $g \equiv 0$.

Proof. The existence of $C$ and the left-hand inequality follow from Izumi's theorem [I, ELS]. Indeed, the latter theorem yields a constant $C>0$ such that $\nu(f) \leq C \nu_{\mathfrak{m}}(f)$ for all functions $f \in R$, hence $\log |\mathfrak{a}|(\nu) \geq C \log |\mathfrak{a}|\left(\nu_{\mathfrak{m}}\right)$ for 
each ideal $\mathfrak{a} \subset R$, and the result follows since every formal psh function is the pointwise limit of functions of this type. The right-hand inequality is the special case of $g \leq g \circ r_{\pi}$ when $\pi$ is the blow-up of $X$ at 0: see Proposition 2.7.

\section{$\S 3.5$. Nef envelopes}

The key technical tool for proving Theorem 3.9 and 3.10 consists of approximating an arbitrary nef Weil divisor by special ones, which are in turn well approximated from below by Weil divisors associated to ideals.

Definition 3.15. If $W$ is a Weil divisor on $\mathfrak{X}$, we let $\mathcal{L}^{\infty}(W)$ be the ideal of functions $f \in \hat{R}$ such that $Z(f) \leq W$.

Clearly $\mathcal{L}^{\infty}(W)$ is an integrally closed ideal in $\hat{R}$, and by definition $Z\left(\mathcal{L}^{\infty}(W)\right) \leq W$. In general, $\mathcal{L}^{\infty}(W)$ may be reduced to 0 . However, there is an important class of Weil divisors for which $\mathcal{L}^{\infty}(W)$ is nontrivial.

Definition 3.16. If $\pi$ is any simple modification, a Weil divisor $W$ on $\mathfrak{X}$ is said to be determined on the dual complex $|\Delta(\pi)|$ if $W=Z(h)$ for some bounded function $h: \mathcal{V} \rightarrow \mathbf{R}$ such that $h=h \circ r_{\pi}$.

For instance any Cartier divisor is determined on a dual complex.

If $W=Z(h)$ is determined on $|\Delta(\pi)|$, then $\mathcal{L}^{\infty}(Z) \neq 0$. Indeed, the condition $Z(f) \leq W$ only needs to be tested on $|\Delta(\pi)|$ by Proposition 2.7 applied to $\log |f|$. But $h$ is bounded so a sufficiently high power of $\mathfrak{m}$ is always included in $\mathcal{L}^{\infty}(W)$.

Since the cone of nef Weil divisors is closed and stable by taking max, the following definition makes sense.

Definition 3.17. $\quad$ For any Weil divisor $W$ on $\mathfrak{X}$ determined on some dual complex, we define the nef envelope $\hat{W}$ as the supremum of all nef Weil divisor $Z$ on $\mathfrak{X}$ such that $Z \leq W$.

If $f \in \hat{R}$ and $t>0, Z(f) \leq t W$ is equivalent to $Z(f) \leq t \hat{W}$ since $t^{-1} Z(f)$ is nef. In other words, we have $\mathcal{L}^{\infty}(t W)=\mathcal{L}^{\infty}(t \hat{W})$ for all $t>0$.

The next result is in essence contained in [ELS], see Remark 3.20 below.

Proposition 3.18. $\quad$ Let $W=\hat{Z}$ be the nef envelope of some Weil divisor $Z$ determined on a dual complex. For $k>0$ set $W_{k}:=\frac{1}{k} Z\left(\mathcal{L}^{\infty}(k W)\right)$. Then there exists a constant $C>0$ such that

(i) $W \leq Z\left(\mathcal{L}^{2}(W)\right) \leq W+A$; 
(ii) $\mathcal{L}^{2}(k W) \subset \mathcal{L}^{\infty}((k-C) W)$ for all $k>C$;

(iii) $W_{k} \leq W \leq(1-C / k) W_{k-C}$ for all $k>C$;

(iv) $\mathcal{L}^{2}(W)=\mathcal{L}^{2}\left(W_{k}\right)$ for $k$ large enough.

Remark 3.19. The proof will show that the constant $C$ in the statement can be taken to be $-\min _{|\Delta(\pi)|} A / g_{W}$ whenever $W$ is determined on $|\Delta(\pi)|$. Note also that (i) is a special case of Theorem 3.9 (which we shall prove in general in Section 3.6.)

Remark 3.20. Pick any simple modification $\pi$, and an effective divisor $D$ in $X_{\pi}$. Then $\mathfrak{a}_{t}=\pi_{*} \mathcal{O}_{X_{\pi}}(-t D)$ satisfies $\mathfrak{a}_{t} \cdot \mathfrak{a}_{s} \subset \mathfrak{a}_{t+s}$, hence defines a graded family of ideals $\mathfrak{a}_{\bullet}$ in the sense of [ELS]. Set $W=\lim _{t} \frac{1}{t} Z\left(\mathfrak{a}_{t}\right)$. Then (iv) above shows that the multiplier ideal of $\mathfrak{a}_{\bullet}$ as defined in op.cit. coincides with $\mathcal{L}^{2}(W)$; whereas (iii) is exactly [ELS, Corollary B].

Remark 3.21. In view of Proposition 3.11 we can similarly define the psh envelope of any function on $\mathcal{V}_{\mathrm{qm}}$ determined on a dual complex. Proposition 3.18 continues to hold in this context.

Proof of Proposition 3.18. Throughout the proof we shall make use of the identification of Weil divisors on $\mathfrak{X}$ with real-valued functions on $\mathcal{V}_{\text {div }}$. The result is trivial when $W=0$ so we shall assume $\nu_{\mathfrak{m}}(W)<0$. We start by proving

Lemma 3.22. With the notation above we have $W=\sup _{k} W_{k}$.

Proof. For any $k, W_{k}$ is nef and $\leq W$ by definition. For the reverse inequality, let $\pi \in \mathfrak{B}$ be large enough, so that $Z$ is determined in $|\Delta(\pi)|$. Since $W$ is nef, given $\varepsilon>0$, there exists by definition a primary ideal $\mathfrak{a}$ of $R$ and an integer $k>0$ such that $W$ and $\frac{1}{k} Z(\mathfrak{a})$ are $\varepsilon$-close on $|\Delta(\pi)| \cap \mathcal{V}_{\text {div }}$. Since $W<0$, we can find an ideal of the form $\mathfrak{b}=\mathfrak{a}^{N} \mathfrak{m}^{M}$ and $l>0$ such that $W-\varepsilon \leq \frac{1}{l} Z(\mathfrak{b}) \leq W$ on $|\Delta(\pi)| \cap \mathcal{V}_{\text {div }}$. A fortiori we have $\frac{1}{l} Z(\mathfrak{b}) \leq Z$ on $|\Delta(\pi)|$, hence everywhere on $\mathcal{V}$ since $Z=r_{\pi}^{*} Z$. This means that $\mathfrak{b} \subset \mathcal{L}^{\infty}(l Z)=$ $\mathcal{L}^{\infty}(l W)$, i.e. $\frac{1}{l} Z(\mathfrak{b}) \leq W_{l} \leq W$. As $\frac{1}{l} Z(\mathfrak{b})$ can be made arbitrarily close to $W$ on $|\Delta(\pi)| \cap \mathcal{V}_{\text {div }}$, we conclude that $W=\sup W_{k}$ on $|\Delta(\pi)| \cap \mathcal{V}_{\text {div }}$ for every large enough $\pi$, hence on all of $\mathcal{V}_{\text {div }}$.

We continue the proof of Proposition 3.18. The right-hand inequality in (i) follows by the definition of $\mathcal{L}^{2}(W)$. On the other hand, as follows from the 
definition, the primary ideal $\mathcal{L}^{\infty}(k W)$ is contained in its usual multiplier ideal $\mathcal{J}\left(\mathcal{L}^{\infty}(k W)\right)$, which is equal to $\mathcal{L}^{2}\left(k W_{k}\right)$ by Proposition 3.7. By the usual subadditivity theorem (Theorem 3.5) we get $\mathcal{L}^{2}\left(k W_{k}\right) \subset \mathcal{L}^{2}\left(W_{k}\right)^{k}$. Moreover, $\mathcal{L}^{2}\left(W_{k}\right) \subset \mathcal{L}^{2}(W)$ since $W_{k} \leq W$. We deduce from this chain of inequalities that $Z\left(\mathcal{L}^{\infty}(k W)\right) \leq k Z\left(\mathcal{L}^{2}(W)\right)$, i.e. $W_{k} \leq Z\left(\mathcal{L}^{2}(W)\right)$, and (i) follows from Lemma 3.22 by letting $k \rightarrow \infty$.

We now prove (ii). Pick $\pi$ such that $Z=r_{\pi}^{*} Z$. If $f \in \mathcal{L}^{2}(k W)$, then $Z(f) \leq k W+A$ by definition. Now we have $A \leq-C W$ on $|\Delta(\pi)|$ for some $C>0$ (which only depends on $\pi$ ) since $W<0$. We infer that $Z(f) \leq(k-$ $C) W \leq(k-C) Z$ on $|\Delta(\pi)|$, hence everywhere since $Z=r_{\pi}^{*} Z$. This means that $f \in \mathcal{L}^{\infty}((k-C) Z)=\mathcal{L}^{\infty}((k-C) W)$ as claimed.

To get (iii), we use (ii). By applying (i) to $k W$ instead of $W$, we get $k W \leq Z\left(\mathcal{L}^{2}(k W)\right) \leq Z\left(\mathcal{L}^{\infty}((k-C) W)\right)$, and the result follows. Finally, to get (iv), first note that $\mathcal{L}^{2}(W)=\mathcal{L}^{2}\left(W+\frac{C}{k} W_{C}\right)$ for $k$ large enough. Now $k W_{k} \leq(k-C) W_{k-C}+C W_{C}$, so $W+\frac{C}{k} W_{C} \leq W_{k}$ by (iii), which concludes the proof.

\section{§3.6. Proofs of Theorems 3.9 and 3.10}

Both proofs proceed by a reduction to the case of nef Weil divisors obtained as nef envelopes of divisors determined on a fixed dual complex.

Proof of Theorem 3.9. Start with an arbitrary nef Weil divisor $W$ on $\mathfrak{X}$. For every $\pi$, we can consider the incarnation $W_{\pi} \in \operatorname{Div}(\mathfrak{X})$ of $W$ on $X_{\pi}$, viewed as a Cartier divisor on $\mathfrak{X}$. In general, $W_{\pi}$ may not be nef; let $\hat{W}_{\pi}$ be its nef envelope. Since $W$ is nef, we have $W \leq \hat{W}_{\pi} \leq W_{\pi}$ by construction.

On the other hand, $W_{\pi}$ and $W$ coincide on the vertices of $\Delta(\pi)$, and it follows that the net $\hat{W}_{\pi}$ decreases pointwise to $W$ as $\pi$ tends to infinity in the directed set of simple modifications. We will rely on the following two results:

Lemma 3.23. Let $\left(Z_{i}\right)_{i \in I}$ be a decreasing net of nef Weil divisors on $\mathfrak{X}$, indexed by a directed set $I$, and set $Z=\inf Z_{i}$. Then $\mathcal{L}^{2}(Z)=\bigcap_{i} \mathcal{L}^{2}\left((1+\varepsilon) Z_{i}\right)$ for every $\varepsilon>0$ small enough.

Lemma 3.24. Let $\mathfrak{a}_{i}$ be a decreasing net of ideals in $\hat{R}$, indexed by a directed set $I$, and set $\mathfrak{a}=\bigcap_{I} \mathfrak{a}_{i}$. Then $\inf _{i \in I} Z\left(\mathfrak{a}_{i}\right)=Z(\mathfrak{a})$.

We deduce from these lemmas that $Z\left(\mathcal{L}^{2}(W)\right)=\lim _{\pi} Z\left(\mathcal{L}^{2}\left((1+\varepsilon) \hat{W}_{\pi}\right)\right)$ for $\varepsilon>0$ small enough. We conclude by applying Proposition 3.18 (i) to $(1+\varepsilon) \hat{W}_{\pi}$ which by definition is the nef envelope of a Cartier divisor. 
Proof of Lemma 3.23. We have $\mathcal{L}^{2}(Z)=\mathcal{L}^{2}((1+\varepsilon) Z)$ for $\varepsilon \ll 1$, hence $\mathcal{L}^{2}(Z) \subset \bigcap_{i} \mathcal{L}^{2}\left((1+\varepsilon) Z_{i}\right)$ since $Z \leq Z_{i}$. Conversely, $f \in \bigcap_{i} \mathcal{L}^{2}\left((1+\varepsilon) Z_{i}\right)$ implies $Z(f) \leq(1+\varepsilon) Z_{i}+A$ for all $i$ hence $f \in \mathcal{L}^{2}(Z)$ since $Z_{i}$ converges to $Z$.

Proof of Lemma 3.24. For every ideal $\mathfrak{b}$ of $\hat{R}, Z(\mathfrak{b})$ is the decreasing limit of $Z\left(\mathfrak{b}+\mathfrak{m}^{k}\right)$ as $k \rightarrow \infty$. Now, because $\hat{R} / \mathfrak{m}^{k}$ has finite length, it is Artinian, so that the decreasing sequence of ideals $\mathfrak{a}_{i}+\mathfrak{m}^{k}$ is stationary for $i \geq i(k)$. We claim that the stationary value is actually $\mathfrak{a}+\mathfrak{m}^{k}$. Granting this for a moment, we get

$$
Z(\mathfrak{a}) \leq \inf _{i \in I} Z\left(\mathfrak{a}_{i}\right) \leq Z\left(\mathfrak{a}_{i(k)}+\mathfrak{m}^{k}\right)=Z\left(\mathfrak{a}+\mathfrak{m}^{k}\right),
$$

and so $Z(\mathfrak{a})=\inf _{i \in I} Z\left(\mathfrak{a}_{i}\right)$ follows by letting $k \rightarrow \infty$. To prove the claim, let $p_{l}: \hat{R} \rightarrow \hat{R} / \mathfrak{m}^{l}$ denote the projection. Each quotient map $\hat{R} / \mathfrak{m}^{l+1} \rightarrow \hat{R} / \mathfrak{m}^{l}$ induces a surjection from $\bigcap_{i \in I} p_{l+1}\left(\mathfrak{a}_{i}\right) \rightarrow \bigcap_{i \in I} p_{l}\left(\mathfrak{a}_{i}\right)$, because both sides are reductions of the same $\mathfrak{a}_{i}$ for $i \in I$ large enough. Because of this, given $f_{k} \in$ $\bigcap_{i \in I} \mathfrak{a}_{i}+\mathfrak{m}^{k}$, there exists $f \in \hat{R}$ such that $f=f_{k} \bmod \mathfrak{m}^{k}$ and $f \in \bigcap_{i \in I}\left(\mathfrak{a}_{i}+\mathfrak{m}^{l}\right)$ for every $l \geq k$. But now $f \in \mathfrak{a}_{i} \bmod \mathfrak{m}^{l}$ for every large $l$ implies $f \in \mathfrak{a}_{i}$ because ideals of $\hat{R}$ are closed by Krull's lemma, and so we get $f \in \bigcap_{i \in I} \mathfrak{a}_{i}=\mathfrak{a}$. This proves the claim and the lemma.

Proof of Theorem 3.10. By the same token, the proof boils down to

Lemma 3.25. Let $\left(\mathfrak{a}_{i}\right)_{i \in I}$ and $\left(\mathfrak{b}_{i}\right)_{i \in I}$ be decreasing nets of ideals in $\hat{R}$, indexed by a directed set $I$. Then $\bigcap_{i}\left(\mathfrak{a}_{i} \mathfrak{b}_{i}\right)=\left(\bigcap_{i} \mathfrak{a}_{i}\right)\left(\bigcap_{i} \mathfrak{b}_{i}\right)$.

Again, we only have to check it modulo $\mathfrak{m}^{l}$ for all $l$ in which case the proof is obvious since the $\mathfrak{a}_{i}$ are stationary modulo $\mathfrak{m}^{l}$.

\section{§4. Intersection Theory and Monge-Ampère Operator}

\section{$\S 4.1$ Intersection of nef Weil divisors}

Fix a modification $\pi \in \mathfrak{B}$. We denote the intersection number of any $n$ tuple of divisors $Z_{1}, \ldots, Z_{n} \in \operatorname{Div}(\pi)$ by $\left\langle Z_{1}, \ldots, Z_{n}\right\rangle \in \mathbf{R}$. If $Z_{1}=\sum_{E} r_{E} E$, then $\left\langle Z_{1}, \ldots, Z_{n}\right\rangle=\sum_{E} r_{E}\left\langle\left. Z_{2}\right|_{E}, \ldots,\left.Z_{n}\right|_{E}\right\rangle$. By expanding further in terms of the coefficients of the other divisors, we see that the intersection product is multilinear and continuous. It is also symmetric in all variables and satisfies the following important monotonicity property: 
Lemma 4.1. Suppose $Z_{i}^{\prime}, Z_{i} \in \operatorname{Div}(\pi)$ are nef divisors satisfying $Z_{i}^{\prime} \leq$ $Z_{i}$. Then

$$
\left\langle Z_{1}^{\prime}, \ldots, Z_{n}^{\prime}\right\rangle \leq\left\langle Z_{1}, \ldots, Z_{n}\right\rangle
$$

Proof. By symmetry, the proof reduces to the case $Z_{i}^{\prime}=Z_{i}$ for $i \geq 2$. But it is then clear, since $\left\langle Z_{1}, \ldots, Z_{n}\right\rangle=\left\langle Z_{1}^{\prime}, \ldots, Z_{n}^{\prime}\right\rangle+\left\langle\left(Z_{1}-Z_{1}^{\prime}\right), Z_{2}^{\prime}, \ldots, Z_{n}^{\prime}\right\rangle$ and $Z_{1}-Z_{1}^{\prime}$ is effective while $Z_{i}^{\prime}$ is nef for $i \geq 2$.

We now extend the previous construction to C-Div $(\mathfrak{X})$. For any collection $Z_{1}, \ldots, Z_{n}$ of Cartier divisors on $\mathfrak{X}$ determined on some $X_{\pi}$, we set

$$
\left\langle Z_{1}, \ldots, Z_{n}\right\rangle:=\left\langle Z_{1, \pi}, \ldots, Z_{n, \pi}\right\rangle .
$$

This definition does not depend on $\pi$, and we have

Proposition 4.2. The intersection product $\mathrm{C}-\mathrm{Div}(\mathfrak{X})^{n} \rightarrow \mathbf{R}$ defined by (4.1) is symmetric, multilinear and continuous for the strong topology. Moreover, if $Z_{i}^{\prime} \leq Z_{i}$ are nef Cartier divisors, then

$$
\left\langle Z_{1}^{\prime}, \ldots, Z_{n}^{\prime}\right\rangle \leq\left\langle Z_{1}, \ldots, Z_{n}\right\rangle \text {. }
$$

Note that $\left\langle Z_{1}, \ldots, Z_{n}\right\rangle \leq 0$ when the $Z_{i}$ are nef, because $Z_{i}$ is then $\leq 0$.

Define the mixed multiplicity $e\left\langle\mathfrak{a}_{1}, \ldots, \mathfrak{a}_{n}\right\rangle$ of a collection of $n$ primary ideals $\mathfrak{a}_{i}$, as $e\left\langle\mathfrak{a}_{1}, \ldots, \mathfrak{a}_{n}\right\rangle:=\operatorname{dim} R /\left(f_{1}, \ldots, f_{n}\right)$ where $f_{i}$ is a generic element in $\mathfrak{a}_{i}$, see also [Re]. Then the following holds, see [Ram]:

$$
-\left\langle Z\left(\mathfrak{a}_{1}\right), \ldots, Z\left(\mathfrak{a}_{n}\right)\right\rangle=e\left\langle\mathfrak{a}_{1}, \ldots, \mathfrak{a}_{n}\right\rangle .
$$

The inequality (4.2) allows us to extend the definition to nef Weil divisors.

Definition 4.3. Let $Z_{1}, \ldots, Z_{n}$ be nef Weil divisors on $\mathfrak{X}$. Then we set

$$
\left\langle Z_{1}, \ldots, Z_{n}\right\rangle:=\inf \left\langle W_{1}, \ldots, W_{n}\right\rangle \in[-\infty, 0],
$$

where the infimum is taken over nef Cartier divisors such that $W_{i} \geq Z_{i}$ for all $i$.

By monotonicity, this definition is consistent with the intersection defined above for nef Cartier divisors.

Proposition 4.4. The intersection product on nef Weil divisors is symmetric, upper semicontinuous, and continuous along decreasing families. It is also 1-homogeneous, additive, and increasing in each variable. Further, $\left\langle Z_{1}, \ldots, Z_{n}\right\rangle<0$ unless $Z_{i}=0$ for some $i$. 
Remark 4.5. Our approximation result Proposition 3.13 whose proof is based on multiplier ideals is used here in an essential way to prove the additivity of the intersection product.

Proof. Symmetry, homogeneity and monotonicity are all clear by definition. Suppose that all the $Z_{i}$ are non-zero. Then there exists $\varepsilon>0$ such that $Z_{i} \leq \varepsilon Z(\mathfrak{m})$ for all $i$ by Proposition 2.4 , and it follows that $\left\langle Z_{1}, \ldots, Z_{n}\right\rangle \leq-\varepsilon^{n}$.

Let us prove upper semicontinuity. The proof relies on the following remark: if $W$ is a Cartier divisor on $\mathfrak{X}$, then the set of nef Weil divisors $Z$ on $\mathfrak{X}$ such that $Z<W$ (coefficient-wise) is open in the nef cone in $\operatorname{Div}(\mathfrak{X})$. Indeed, this condition needs only be tested on the finitely many exceptional primes of a determination $\pi$ of $W$ by Proposition 2.4. Now let $Z_{1}, \ldots, Z_{n}$ be given nef Weil divisors. Since the intersection product of nef Weil divisors is always nonpositive, upper semicontinuity at $Z_{1}, \ldots, Z_{n}$ is automatic if one the $Z_{i}$ is zero. We can therefore assume that they are all non-zero, so that $\left\langle Z_{1}, \ldots, Z_{n}\right\rangle<0$ by what precedes.

Let $t<0$ be a given real number such that $\left\langle Z_{1}, \ldots, Z_{n}\right\rangle<t$. By definition, there exist nef Cartier divisors $W_{i} \geq Z_{i}$ such that $\left\langle W_{1}, \ldots, W_{n}\right\rangle<t$. The $W_{i}$ are in particular non-zero, thus $W_{i}<0$ coefficient-wise by Proposition 2.4. Therefore, upon replacing $W_{i}$ by $(1-\varepsilon) W_{i}$ for $\varepsilon \ll 1$ we can assume that $Z_{i}<W_{i}$ by the remark above. Thus the set $U_{i}$ of nef Weil divisors $Z_{i}^{\prime}$ such that $Z_{i}^{\prime}<W_{i}$ is a neighborhood of $Z_{i}$ in the nef cone, and $\left\langle Z_{1}^{\prime}, \ldots, Z_{n}^{\prime}\right\rangle \leq$ $\left\langle W_{1}, \ldots, W_{n}\right\rangle<t$ for $Z_{i}^{\prime}$ in $U_{i}$. This proves upper semi-continuity. Since the intersection product is increasing in each variable, continuity along decreasing families follows immediately.

As to additivity, let $Z_{1}^{\prime}$ be another nef Weil divisor. By Proposition 3.13, there exists decreasing sequences $\left(W_{1, k}\right)_{k=1}^{\infty},\left(W_{1, k}^{\prime}\right)_{k=1}^{\infty}$ and $\left(W_{i, k}\right)_{k=1}^{\infty}, i \geq 2$ of nef Cartier divisors on $\mathfrak{X}$, converging to $Z_{1}, Z_{1}^{\prime}$ and $Z_{i}$ respectively. Thus additivity follows from the continuity just proved and from the additivity of the intersection pairing on Cartier divisors.

\section{§4.2. Monge-Ampère measures on $\mathcal{V}$}

We now interpret the intersection product on nef Weil divisors constructed above as a positive measure on $\mathcal{V}$.

Recall that a Radon measure on $\mathcal{V}$ is a continuous linear form on the space $C^{0}(\mathcal{V})$ of continuous real-valued functions on $\mathcal{V}$ (with respect to its weak topology), endowed with the sup norm. If $W$ is a dense vector subspace of $C^{0}(\mathcal{V})$ that contains the constant functions, then any non-negative linear form 
$L$ on $W$ defines a positive Radon measure on $\mathcal{V}$, of total mass $L(\mathbf{1})$ with $\mathbf{1}$ the constant function on $\mathcal{V}$. Indeed, for every $f \in W$, we have $(\inf f) \mathbf{1} \leq$ $f \leq(\sup f) \mathbf{1}$, and thus $(\inf f) L(\mathbf{1}) \leq L(f) \leq(\sup f) L(\mathbf{1})$. In other words $|L(f)| \leq L(\mathbf{1}) \sup |f|$, and the result follows by uniform continuity.

Proposition 4.6. Suppose $g_{1}, \ldots, g_{n-1}$ are formal psh functions on $\mathcal{V}$, with associated nef divisors $Z_{i}$ on $\mathfrak{X}$. If $\left\langle Z_{1}, \ldots, Z_{n-1}, Z(\mathfrak{m})\right\rangle>-\infty$, there exists a unique positive Radon measure $\mathrm{MA}\left(g_{1}, \ldots, g_{n-1}\right)$ on $\mathcal{V}$ such that

$$
\int_{\mathcal{V}} g \operatorname{MA}\left(g_{1}, \ldots, g_{n-1}\right)=\left\langle Z(g), Z_{1}, \ldots, Z_{n-1}\right\rangle,
$$

for any formal psh function $g$ on $\mathcal{V}$.

When $\left\langle Z_{1}, \ldots, Z_{n-1}, Z(\mathfrak{m})\right\rangle>-\infty$ holds, we will say that the MongeAmpère measure $\operatorname{MA}\left(g_{1}, \ldots, g_{n-1}\right)$ is well-defined. This is the case if the $g_{i}$ are bounded on $\mathcal{V}$. Indeed, $g_{i} \geq-C$ means $Z_{i} \geq C Z(\mathfrak{m})$, thus $\left\langle Z_{1}, \ldots, Z_{n-1}, Z(\mathfrak{m})\right\rangle$ $\geq-C^{n-1}$ by monotonicity.

Proof. If $W$ is a given nef Cartier divisor on $\mathfrak{X}$, with associated continuous (and piecewise affine) function $g_{W}$ on $\mathcal{V}$, then $g_{W}$ is bounded on the compact space $\mathcal{V}$, i.e. $g_{W} \geq-C$ for some $C>0$. In terms of divisors, this means that $W \geq C Z(\mathfrak{m})$, and thus our assumption implies that $\left\langle Z_{1}, \ldots, Z_{n-1}, W\right\rangle>-\infty$ for any nef Cartier divisor $W$. Now if $Z$ is an arbitrary Cartier divisor on $\mathfrak{X}$, we can write it as a difference of two nef Cartier divisors $Z=W_{1}-W_{2}$, and we then set $\left\langle Z_{1}, \ldots, Z_{n-1}, Z\right\rangle:=\left\langle Z_{1}, \ldots, Z_{n-1}, W_{1}\right\rangle-\left\langle Z_{1}, \ldots, Z_{n-1}, W_{2}\right\rangle$. This does not depend on the choice of $W_{1}$ and $W_{2}$ by additivity. We thus get a non-negative functional $Z \mapsto\left\langle Z_{1}, \ldots, Z_{n-1}, Z\right\rangle$ on $\mathrm{C}-\operatorname{Div}(\mathfrak{X})$. By Proposition 1.14, C-Div $(\mathfrak{X})$ can be identified with the dense subspace $\operatorname{PA}(\mathcal{V})$ of $C^{0}(\mathcal{V})$, see Proposition 1.15. By the preceding discussion, this concludes the proof.

By translating Proposition 4.4, (4.2) and (4.3) into statements about psh functions we obtain the following two results.

Proposition 4.7. For any primary ideals $\mathfrak{a}_{1}, \ldots, \mathfrak{a}_{n}$ of $R$, we have

$$
e\left\langle\mathfrak{a}_{1}, \ldots, \mathfrak{a}_{n}\right\rangle=\int_{\mathcal{V}}-\log \left|\mathfrak{a}_{1}\right| \mathrm{MA}\left(\log \left|\mathfrak{a}_{2}\right|, \ldots, \log \left|\mathfrak{a}_{n}\right|\right) .
$$

Proposition 4.8. Suppose $\left(g_{i, k}\right)_{k=1}^{\infty}$ is a sequence of formal psh functions decreasing to a formal psh function $g_{i}$ for $i=1, \ldots, n-1$. If $\rho:=$ $\operatorname{MA}\left(g_{1}, \ldots, g_{n-1}\right)$ is defined, then so is $\rho_{k}:=\operatorname{MA}\left(g_{1, k}, \ldots, g_{n-1, k}\right)$ for all $k$, and $\rho_{k} \rightarrow \rho$ weakly as $k \rightarrow \infty$ 
Let us spell out more explicitly what happens for piecewise affine functions.

Proposition 4.9. If $g_{1}, \ldots, g_{n-1}$ are piecewise affine formal psh functions on $\mathcal{V}$, then $\operatorname{MA}\left(g_{1}, \ldots, g_{n-1}\right)$ is a finite sum of Dirac masses at divisorial valuations.

Proof. Choose $\pi$ such that each $g_{i}$ belongs to $\operatorname{Aff}(\pi)$, and let $g$ be a piecewise affine test function, lying in $\operatorname{Aff}\left(\pi^{\prime}\right)$ for some $\pi^{\prime}$ possibly very large. By definition, we then have $\int_{\mathcal{V}} g \mathrm{MA}\left(g_{1}, \ldots, g_{n-1}\right)=\left\langle Z(g), Z\left(g_{1}\right), \ldots, Z\left(g_{n-1}\right)\right\rangle$, which can be computed on $X_{\pi}$ itself by the projection formula, even if $Z(g)$ is only determined by $\pi^{\prime}$. The integral is thus equal to $\sum_{E} g\left(\nu_{E}\right) b_{E}\left\langle E, Z\left(g_{1}\right), \ldots\right.$, $\left.Z\left(g_{n-1}\right)\right\rangle$, where the sum is over the vertices of $\Delta(\pi), \nu_{E}=b_{E}^{-1} \operatorname{ord}_{E}$ is the normalized valuation proportional to $\operatorname{ord}_{E}$, and $b_{E}=\operatorname{ord}_{E}(\mathfrak{m})$ is the normalization factor. We thus see that $\mathrm{MA}\left(g_{1}, \ldots, g_{n-1}\right)$ is supported on the vertices of $\Delta(\pi)$, with mass $b_{E}\left\langle E, Z\left(g_{1}\right), \ldots, Z\left(g_{n-1}\right)\right\rangle$ at the vertex $\nu_{E}$ determined by a $\pi$-exceptional prime $E$.

In fact, this reasoning shows that the intersection number $\left\langle Z_{1}, \ldots, Z_{n}\right\rangle$ of nef Weil divisors on $\mathfrak{X}$ is always finite when all but at most one $Z_{i}$ are Cartier. Equivalently, the Monge-Ampère measure $\mathrm{MA}\left(g_{1}, \ldots, g_{n-1}\right)$ of formal psh functions is always well-defined when all but at most one of the $g_{i}$ are piecewise affine.

In the case $n=2$, we thus see that $\operatorname{MA}(g)$ is always defined when $g$ is a formal psh function on the valuative tree $\mathcal{V}$. One can check that $\mathrm{MA}(g)$ coincides with the Laplacian $\Delta(-g)$ of the (positive) tree potential $-g$ as defined in [FJ1]. In this special case, the Laplace operator can be constructed purely in terms of the metric tree structure of $\mathcal{V}$. See also [BR], [T1]. In the higher dimensional case, the metric tree structure is replaced by the piecewise integral structure on $\mathcal{V}$ introduced in Section 1.6, but this is not enough to define the Monge-Ampère operator.

\section{$\S 5 . \quad$ Singularities of Plurisubharmonic Functions}

Throughout this section, we work over the field of complex numbers, and denote by $\mathcal{O}$ the ring of germs of holomorphic functions at the origin in $\mathbf{C}^{n}$. It lies between the two rings $R \subset \hat{R}$ we have been considering, and primary ideals of all three rings can (and will) be identified. Given an analytic ideal $\mathfrak{a} \subset \mathcal{O}$ and a valuation $\nu \in \hat{\mathcal{V}}$, we will compute $\nu(\mathfrak{a})$ as the value of $\nu$ on the formal ideal $\mathfrak{a} \hat{R}$. 


\section{$\S 5.1 . \quad$ Definitions}

Let $u$ be a psh germ, that is, a plurisubharmonic function defined in a neighborhood of the origin in $\mathbf{C}^{n}$. The origin is a singularity of $u$ if $u(0)=-\infty$. When $u$ and $v$ are two psh germs, we say that $u$ is more singular than $v$ if $u \leq v+O(1)$ near 0 . We say that $u$ has singularities described by $\mathfrak{a}^{c}$ for some ideal $\mathfrak{a}$ of $\mathcal{O}$ and $c>0$ if $u=c \max \log \left|f_{k}\right|+O(1)$ for some (hence any) choice of local generators $\left(f_{k}\right)$ of $\mathfrak{a}$.

Recall that the Lelong number $\nu^{\mathrm{L}}(u, 0) \in \mathbf{R}_{+}$of $u$ is the supremum of all $c \geq 0$ such that $u$ is more singular than $c \log |z|$. The supremum is actually attained, as follows from the basic fact that $\sup _{\{\log |z|<t\}} u$ is a convex function of $t$. When $u$ has singularities described by an analytic ideal $\mathfrak{a}, \nu^{\mathrm{L}}(u, 0)$ is just $\nu_{\mathfrak{m}}(\mathfrak{a})$.

The multiplier ideal $\mathcal{J}(u) \subset \mathcal{O}$ of a psh germ $u$ is the ideal of germs of holomorphic functions $f$ such that $f e^{-u}$ is $L^{2}$ near 0 . When the singularity of $u$ is described by $\mathfrak{a}^{c}$, it is elementary to see that this definition coincides with the one given in Section 3.2: see e.g. [DK, Proposition 1.7].

For any psh germ, the multiplier ideals $\mathcal{J}(k u)$ allow us to approximate the singularity of $u$ very precisely as $k \rightarrow \infty$. We refer to [DK, Theorem 4.2] for details on the following construction. Fix a ball $B$ centered at the origin on which $u$ is defined, and let $\mathcal{H}(u, B)$ be the set of holomorphic functions $f$ on $B$ such that $\|f\|^{2}:=\int_{B}|f|^{2} e^{-2 u}<+\infty$. This is a Hilbert space, and one can show that the germs at 0 of the functions of $\mathcal{H}(u, B)$ generate $\mathcal{J}(u)$. This implies that the psh germ $\mathcal{R} u:=\sup \{\log |f|, f \in \mathcal{H}(u, B),\|f\|=1\}$ on $B$ has singularities described by $\mathcal{J}(u)$ at 0 .

Now it is a fundamental fact that there exists a constant $C>0$ depending only on the dimension $n$ and the diameter of $B$, such that

$$
u(p)-C \leq \mathcal{R} u(p) \leq \sup _{B(p, r)} u+C-n \log r,
$$

for all $p$ and all $r$ such that $B(p, r) \subset B$. Here the right hand side is a consequence of the mean value inequality for $|f|^{2}$ when $f$ is holomorphic, whereas the left hand-side is a deep fact, an application of the Ohsawa-Takegoshi theorem.

Using (5.1), it is easy to see that $u_{k}:=\frac{1}{k} \mathcal{R}(k u)$ converges pointwise to $u$ as $k \rightarrow \infty$, and that $\frac{1}{k} \nu_{\mathfrak{m}}(\mathcal{J}(k u))=\nu^{\mathrm{L}}\left(u_{k}, 0\right)$ converges to $\nu^{\mathrm{L}}(u, 0)$. We will now show that this convergence extends to $\mathcal{V}$.

\section{§5.2. Valuative transform of a psh germ}

We can associate to a psh germ $u$ a Weil divisor $Z(u) \in \operatorname{Div}(\mathfrak{X})$ as follows. If $E$ is an exceptional prime on some $X_{\pi}$, we define $-\operatorname{ord}_{E} Z(u)$ to be the 
generic Lelong number of $u \circ \pi$ along $E$, i.e. its Lelong number at the very general point of $E$. This only depends on the divisorial valuation $\operatorname{ord}_{E}$. Equivalently, $\left[Z(u)_{\pi}\right]$ is the divisorial part in the Siu decomposition of the current $d d^{c}(u \circ \pi)$ on $X_{\pi}$.

Theorem 5.1. For any psh germ $u$, the Weil divisor $Z(u)$ on $\mathfrak{X}$ is nef.

Definition 5.2. We write $\hat{u}$ for the associated formal psh function on $\mathcal{V}$ and call it the valuative transform of $u$.

Let us relate the analytic multiplier ideal $\mathcal{J}(u)$ of $u$ to its valuative counterpart $\mathcal{L}^{2}(\hat{u})$. For that purpose, note that the family of ideals $\mathcal{J}((1+\varepsilon) u)$ increases as $\varepsilon>0$ decreases to 0 , thus has to be stationary by the Noetherian property. This justifies the following definition.

Definition 5.3. For any psh germ $u$, we set $\mathcal{J}_{+}(u):=\mathcal{J}((1+\varepsilon) u)$ for $\varepsilon>0$ sufficiently small.

Remark 5.4. The Openness Conjecture by Demailly and Kollár [DK, Remark 5.3] asserts that $\mathcal{J}_{+}(u)=\mathcal{J}(u)$ for any psh germ $u$. In [FJ3] this conjecture was settled affirmatively in dimension two.

Theorem 5.5. If $u$ is any psh germ, then we have $\mathcal{L}^{2}(\hat{u}) \cap \mathcal{O}=\mathcal{J}_{+}(u)$.

Let us give an explicit interpretation of the restriction of the valuative transform $\hat{u}$ to a simplex in $\mathcal{V}$. Let $\pi$ be a simple modification, and $E_{1}, \ldots, E_{k}$ be exceptional primes of $\pi$ determining a simplex $|\sigma|$ in $|\Delta(\pi)| \subset \mathcal{V}$. Let $z_{1}, \ldots, z_{k}$ be local equations of $E_{1}, \ldots, E_{k}$ at a generic point $p$ on $E_{1} \cap \cdots \cap E_{k}$, and complete these to local coordinates $z_{1}, \ldots, z_{n}$ at $p$. Then $|\sigma|$ consists of those valuations $\nu_{z, w}, w \in \mathbf{R}_{+}^{n}$ that are monomial in this coordinate system, and such that $\sum_{1 \leq i \leq k} b_{i} w_{i}=1, w_{i}=0$ for $i>k$. Here $b_{i}=\operatorname{ord}_{E_{i}}(\mathfrak{m})$. We will identify $\sigma$ with the set of such $w \in \mathbf{R}_{+}^{n}$.

Now for any $w \in \mathbf{R}_{+}^{n}$, if $v$ is a psh function on $X_{\pi}$ near $p$, set

$$
\nu_{z, w}^{\mathrm{K}}(v)=\sup \left\{t \geq 0, v \leq t \log \max \left|z_{i}\right|^{w_{i}}+O(1)\right\} .
$$

This weighted version of the Lelong number was introduced in [Ki] and is referred to as the Kiselman number. Kiselman numbers are 1-homogeneous and monotonous in $w: \nu_{z, c w}^{\mathrm{K}}=c \nu_{z, w}^{\mathrm{K}}$ for any $c>0$; and $w_{i} \leq w_{i}^{\prime}$ for all $i$ implies $\nu_{z, w}^{\mathrm{K}}(v) \geq \nu_{z, w^{\prime}}^{\mathrm{K}}(v)$. In particular, the function $w \mapsto \nu_{z, w}^{\mathrm{K}}(v)$ is continuous in $w$.

When $w \in \mathbf{Q}_{+}^{n}$, the monomial valuation $\nu_{z, w} \in|\sigma|$ is divisorial so we may write $\nu_{z, w}=c \operatorname{ord}_{E}$ for some exceptional divisor $E$ of a modification $\mu$ of $X_{\pi}$ 
above $\pi^{-1}(0)$, and some positive real number $c>0$. It is then not difficult to see that $\nu_{z, w}^{\mathrm{K}}(v)=c \operatorname{ord}_{E}(v \circ \mu)$. If $v=u \circ \pi$, this shows $\hat{u}\left(\nu_{z, w}\right)=-\nu_{z, w}^{\mathrm{K}}(u \circ \pi)$ when $w \in \mathbf{Q}_{+}^{n}$. By continuity, we conclude

Proposition 5.6. For any simple modification $\pi$, and any quasimonomial valuation $\nu_{z, w}$ in a simplex $|\sigma| \subset \mathcal{V}$ appearing as a face on $|\Delta(\pi)|$, and any psh germ $u$, the number $\hat{u}\left(\nu_{z, w}\right)$ equals the Kiselman number $-\nu_{z, w}^{\mathrm{K}}(u \circ \pi)$.

In particular, the Kiselman numbers are concave functions of the weight $w$, see Proposition 3.11. This fact is due to Kiselman. From [D2, Proposition 3.12], one also infers

Corollary 5.7. $\quad$ Let $u_{j}, u$ be psh germs such that $u_{j} \rightarrow u$ in $L_{\mathrm{loc}}^{1}$. Then

$$
\liminf _{j \rightarrow \infty} \hat{u}_{j}(\nu) \geq \hat{u}(\nu)
$$

for any quasimonomial valuation $\nu$.

Proof of Theorem 5.1. By what was recalled above, $u$ is more singular than the psh germ $\mathcal{R}(u)$, which has a singularity described by $\mathcal{J}(u)$. Hence $Z(u) \leq Z(\mathcal{J}(u))$. On the other hand, let $f \in \mathcal{J}(u)$. If $\pi \in \mathcal{B}$ and $E$ is an exceptional prime of $\pi$, the local integrability of $|f|^{2} e^{-2 u}$ implies the integrability of $|f \circ \pi|^{2} e^{-2 u \circ \pi}|J \pi|^{2}$ on $X_{\pi}$ by the change of variables formula, and this in turn is easily seen to imply $\operatorname{ord}_{E}(f \circ \pi)+A\left(\operatorname{ord}_{E}\right)>\operatorname{ord}_{E}(u \circ \pi)$, as a consequence of Fubini's formula at a generic point of $E$. It follows that $\operatorname{ord}_{E}(\mathcal{J}(u))+A\left(\operatorname{ord}_{E}\right) \geq \operatorname{ord}_{E}(u)$, and we conclude

$$
Z(\mathcal{J}(u)) \geq Z(u) \geq Z(\mathcal{J}(u))-A
$$

It follows that $Z(u)$ is the limit of the nef divisors $\frac{1}{k} Z(\mathcal{J}(k u))$, hence is nef.

Proof of Theorem 5.5. If $f \in \mathcal{J}_{+}(u)$, then we have $Z(f) \leq Z((1+\varepsilon) u)+A$ for $\varepsilon>0$ small enough by (5.3), and this implies that $f \in \mathcal{L}^{2}(\hat{u})$ by definition.

In case $u$ has singularities described by $\mathfrak{a}^{c}$ for some analytic ideal $\mathfrak{a}$ of $\mathcal{O}$ (not necessarily primary), the reverse inclusion is proved exactly as in Proposition 3.7, using an analytic log-resolution of $\mathfrak{a}$.

Now let $u$ be an arbitrary psh germ, defined on a small ball $B$ centered at 0 , and let $u_{k}=\frac{1}{k} \mathcal{R}(k u)$, so that $u_{k}$ has singularities described by $\mathcal{J}(k u)^{1 / k}$. We have $\mathcal{L}^{2}(\hat{u})=\mathcal{L}^{2}((1+\varepsilon) \hat{u})$ for small $\varepsilon>0$ by definition, and $\mathcal{L}^{2}((1+\varepsilon) \hat{u}) \subset$ $\mathcal{L}^{2}\left((1+\varepsilon) \hat{u}_{k}\right)$ for all $k$ since $u_{k}$ is less singular than $u$. By the special case just considered, we have $\mathcal{L}^{2}\left((1+\varepsilon) \hat{u}_{k}\right) \cap \mathcal{O}=\mathcal{J}\left((1+\varepsilon) u_{k}\right)$. Thus if $f \in \mathcal{L}^{2}(\hat{u}) \cap \mathcal{O}$, 
we have $\int|f|^{2} e^{-2(1+\varepsilon) u_{k}}<+\infty$ for all $k$. On the other hand, $u_{k}$ satisfies $\int e^{2 k\left(u_{k}-u\right)}<+\infty$, and these two inequalities imply $\int|f|^{2} e^{-2 u}<+\infty$ by the Hölder inequality, as in [DK, Proof of Theorem 4.2]. This concludes the proof.

\section{$\S 5.3$. Weights}

By a psh weight, we will mean a psh germ $\varphi$ having an isolated singularity at the origin and such that $e^{\varphi}$ is continuous.

We now introduce a class of psh germs that will play an important role in the sequel.

Definition 5.8. A psh germ $\varphi$ is said to be tame if there exists a constant $C>0$ such that for every $t>0$ and every germ $f \in \mathcal{J}(t \varphi), \log |f|$ is more singular than $(t-C) \varphi$.

The definition can be paraphrased by saying that the $L^{2}$ and $L^{\infty}$ conditions for holomorphic functions with respect to $e^{-t \varphi}$ stay uniformly bounded as $t \rightarrow$ $\infty$. Concretely, we have the following lemma.

Lemma 5.9. Suppose $\varphi$ is a tame psh germ, and $\varphi_{k}$ is any psh function with singularities described by $\frac{1}{k} \mathcal{J}(k \varphi)$. Then $\varphi \leq \varphi_{k} \leq(1-C / k) \varphi$ up to bounded functions.

Proof. It is sufficient to prove the lemma for $\varphi_{k}:=\frac{1}{k} \mathcal{R}(k \varphi)$. From (5.1), we get $\varphi \leq \varphi_{k}+O(1)$. Conversely, pick a finite set of generators $f_{i}$ of the ideal $\mathcal{J}(k \varphi)$. Then $\varphi_{k}=\frac{1}{k} \log \max \left|f_{i}\right|+O(1)$ near the origin and from the definition of tame weight we have $\log \left|f_{i}\right| \leq(k-C) \varphi$ up to a constant.

We shall be mostly concerned with tame weights. Here is an important class of such weights.

Lemma 5.10. Let $\varphi$ be a psh germ such that $e^{\varphi}$ is $\alpha$-Hölder for some $\alpha>0$. Then $\varphi$ is a tame weight. In fact, we can take $C=n / \alpha$ in the above definition.

Proof. Assume that $\varphi$ is defined on a ball B. By (5.1) we have $\frac{1}{k} \mathcal{R}(k \varphi)(p)$ $\leq \sup _{B(p, r)} \varphi-n \log r+C$ for every $r$ small enough and some uniform constant $C>0$. Now $e^{\varphi}$ is $\alpha$-Hölder, thus $\sup _{B(p, r)} e^{\varphi} \leq e^{\varphi(p)}+C r^{\alpha}$. Choosing $r=e^{\varphi(p) / \alpha}$ now shows that $\sup _{B(p, r)} \varphi \leq \varphi(p)+O(1)$, and this concludes the proof. 
Remark 5.11. One can export the notion of tameness into $\mathcal{V}$. Define a formal psh function $g$ on $\mathcal{V}$ to be tame when $\mathcal{L}^{2}(k g) \subset \mathcal{L}^{\infty}((k-C) g)$ for some $C>0$ and all $k$, or equivalently $g \leq h_{k} \leq(1-C / k) g$ with $h_{k}:=\frac{1}{k} \log \left|\mathcal{L}^{2}(k g)\right|$. In particular, $\mathcal{L}^{\infty}(\mathrm{kg})$ is non-trivial, and in fact $g_{k}:=\frac{1}{k} \log \left|\mathcal{L}^{\infty}(k g)\right|$ converges to $g$ as $k \rightarrow \infty$. Proposition 3.18 and Remark 3.21 assert that the formal psh envelope of a function determined on a dual complex is tame, and the rest of Proposition 3.18 in fact holds for any tame formal psh function.

\section{§5.4. Relative types and valuations}

Let us say that a psh weight $\varphi$ is maximal if it is maximal outside the origin, that is, $\left(d d^{c} \varphi\right)^{n}=\lambda \delta_{0}$ for some $\lambda>0$. If $u$ is an arbitrary psh germ, Rashkovskii [Ras1] defined the relative type $\sigma(u, \varphi)$ of $u$ with respect to $\varphi$ as the largest $c \geq 0$ such that $u$ is more singular than $c \varphi$. It is a fundamental fact that $\sup _{\{\varphi \leq t\}} u$ is a convex function of $t$ under the maximality assumption. Hence $\sigma(u, \varphi)<+\infty$ and that $u$ is more singular than $\sigma(u, \varphi) \varphi$.

We now relate quasi-monomial valuations to relative types with respect to tame maximal weights.

Theorem 5.12. If $\varphi$ is a tame maximal weight and $u$ is any psh germ, then $u \leq \varphi+O(1)$ iff $\hat{u} \leq \hat{\varphi}$. In other words for every psh germ $u$, we have $\sigma(u, \varphi)=\sup \{c \geq 0, \hat{u} \leq c \hat{\varphi}\}$.

Theorem 5.13. For any quasi-monomial valuation $\nu \in \mathcal{V}$, there exists a tame maximal psh weight $\varphi$ such that $\hat{u}(\nu)=\sigma(u, \varphi)$ for any psh function $u$.

The weight $\varphi$ is unique up to a bounded function, and $\hat{\varphi}$ can be characterized as the largest formal psh function $g$ on $\mathcal{V}$ such that $g(\nu)=-1$.

Remark 5.14. The proof of Theorem 5.13 shows that the constant $C$ making $\varphi$ tame can be taken to be $A(\nu)$, and one can show that this is the best possible choice. Also a substantial part of the result is a direct consequence of [Ras1, Theorem 4.1]. Our main contribution lies in proving that $\varphi$ is continuous and tame.

Proof of Theorem 5.12. If $u$ is more singular than $\varphi$, then of course $\hat{u} \leq$ $\hat{\varphi}$. To get the converse, let $\varphi_{k}$ be a psh germ with singularities described by $\mathcal{J}(k \varphi)^{1 / k}$. If $\hat{u} \leq \hat{\varphi}$, then a fortiori $\hat{u} \leq \frac{1}{k} \log |\mathcal{J}(k \varphi)|$. Pick a $\log$-resolution $\pi_{k}$ of the primary ideal $\mathcal{L}^{2}(k \varphi)$, so that $\varphi_{k} \circ \pi_{k}$ has divisorial singularities. Then we have

$$
\operatorname{ord}_{E}\left(u \circ \pi_{k}\right)=-\hat{u}\left(\operatorname{ord}_{E}\right) \geq-\frac{1}{k} \log |\mathcal{J}(k \varphi)|\left(\operatorname{ord}_{E}\right)=\operatorname{ord}_{E}\left(\varphi_{k} \circ \pi_{k}\right)
$$


for each exceptional prime $E$ of $\pi_{k}$, and it follows from a well-known theorem of Siu that $u \circ \pi_{k}-\varphi_{k} \circ \pi_{k}$ is psh up to a bounded term. It is in particular bounded from above, and we infer that $u \leq \varphi_{k}+O(1)$ near the origin. Now Lemma 5.9 implies that $u \leq(1-C / k) \varphi+O(1)$, i.e. $\sigma(u, \varphi) \geq 1-C / k$ for all $k$, and we conclude that $\sigma(u, \varphi) \geq 1$, i.e. $u \leq \varphi+O(1)$.

Proof of Theorem 5.13. We may suppose we work on the unit ball $B$, and define $\varphi$ using a classical envelope construction:

$$
\varphi=\sup \{u \leq 0 \operatorname{psh} \text { on } B \text { such that } \hat{u}(\nu) \leq-1\} .
$$

Choquet's lemma gives a sequence of negative psh functions $v_{j}$ with $\hat{v}_{j}(\nu) \leq-1$ and increasing to a function $v$ whose upper-semicontinuous regularization $v^{*}$ is a psh function equal to $\varphi^{*} \geq \varphi$. From (5.2), we infer $\hat{v}^{*}(\nu) \leq \liminf \hat{v}_{j}(\nu) \leq-1$. This shows $v^{*} \leq \varphi$, hence $\varphi=\varphi^{*}$ is psh.

As $\log |z|$ is a negative psh function whose valuative transform is constant equal to -1 , we get $\log |z| \leq \varphi$ and $\hat{\varphi}(\nu)=-1$. It is not difficult to see that $\log |z|$ is also the largest negative psh function on $B$ such that $\hat{u}\left(\nu_{\mathfrak{m}}\right) \leq-1$. Whence $\log |z| \leq \varphi \leq c \log |z|$ with $c=-\hat{\varphi}\left(\nu_{\mathfrak{m}}\right)$. This implies $\varphi$ to be locally bounded outside 0 and $e^{\varphi}$ to be continuous at 0 . Maximality of $\varphi$ follows from standard arguments.

We now show that

$$
\hat{\varphi}=\sup \{h \text { formal psh, } h(\nu) \leq-1\} \text {. }
$$

Denote by $g$ the right hand side of (5.4). As $\hat{\varphi}$ is a formal psh function with $\hat{\varphi}(\nu)=-1$, we have $\hat{\varphi} \leq g$. Set $g_{k}=\frac{1}{k} \mathcal{L}^{\infty}(k g)$. We claim that $g_{k} \rightarrow g$. To see that, pick a simple blow-up $\pi$ such that $\nu \in|\Delta(\pi)|$. Define the bounded function $h: \mathcal{V} \rightarrow \mathbf{R}$ by setting $h(\nu)=-1, h \equiv 0$ on $|\Delta(\pi)| \backslash\{\nu\}$ and $h=h \circ r_{\pi}$. Then $g$ is the formal psh function associated to the nef envelope of $Z(h)$ so that we may apply Proposition 3.18. We conclude $g_{k} \rightarrow g$.

Now the ideals $\mathcal{L}^{\infty}(k g)$ are primary. One can hence find a negative psh function $u_{k}$ on $B$ with singularities described by $\frac{1}{k} \mathcal{L}^{\infty}(k g)$. As $g_{k} \rightarrow g$, we get $(1+\varepsilon) u_{k} \leq \varphi$ for large $k$ and $\varepsilon$ arbitrarily small. Then $(1+\varepsilon) g_{k} \leq \hat{\varphi}$ and thus $g \leq \hat{\varphi}$. This proves (5.4).

Next we show the continuity of $e^{\varphi}$. The argument is standard. By what precedes, we only need to check the continuity of $\varphi$ outside 0 . Write $\varphi_{k}=$ $\frac{1}{k} \mathcal{R}(k \varphi)$. We shall prove that $\varphi_{k} \rightarrow \varphi$ uniformly on any compact set in $B \backslash\{0\}$. Fix $\varepsilon>0$ arbitrarily small. From (5.1), one infers $\sup \left\{\varphi_{k},|z| \leq 1-\varepsilon\right\} \leq \varepsilon$ and $\varphi_{k} \geq \varphi-\varepsilon$ for $k$ large enough. Set $\bar{\varphi}_{k}=\max \left\{2 \log |z|, \varphi_{k}-4 \varepsilon\right\}$ on $\{|z| \leq 1-\varepsilon\}$ 
and $\bar{\varphi}_{k}=2 \log |z|$ on $\{1-\varepsilon \leq|z| \leq 1\}$. As $\varphi_{k} \geq \varphi-\varepsilon \geq \log |z|-\varepsilon, \bar{\varphi}_{k}$ defines a psh function equal to $\varphi_{k}-4 \varepsilon$ on the fixed ball $\left\{|z| \leq e^{-5 \varepsilon}\right\}$. Look at $\bar{\varphi}_{k}+\varepsilon \log |z|$ : it is a negative psh function on $B$ whose valuative transform equals $\hat{\varphi}_{k}-\varepsilon$. For $k$ sufficiently large, we get $\hat{\varphi}_{k}(\nu)-\varepsilon \leq-1$, and we conclude that $\varphi_{k}+\varepsilon \log |z| \leq \varphi \leq \varphi_{k}+\varepsilon$ on $\left\{|z| \leq e^{-5 \varepsilon}\right\}$ for all $k$ large. This shows uniform convergence on compact subsets of $\varphi_{k} \rightarrow \varphi$ as required.

We have shown that $\varphi$ is a maximal psh weight. Pick a smaller ball $B^{\prime} \subset$ $B$, and set $\varphi^{\prime}=\sup \left\{u \leq 0\right.$ psh on $B^{\prime}$ such that $\left.\hat{u}(\nu) \leq-1\right\}$. The previous argument shows that $\varphi$ is a psh weight, $\varphi \geq \log |z|+O(1)$, and $\hat{\varphi}^{\prime}=\hat{\varphi}$. For suitable constants, the psh function $\max \left\{\varphi^{\prime}-C_{1}, C_{2} \log |z|\right\}$ extends as a negative psh function on $B$, equal to $\varphi^{\prime}-C_{1}$ near 0 . It is thus dominated by $\varphi$. The reverse inequality $\varphi \leq \varphi^{\prime}$ is clear by construction. We conclude that the difference $\varphi-\varphi^{\prime}$ is bounded on $B^{\prime}$.

Fix a psh germ $u$ defined in a ball $B^{\prime}$. Then $u \leq-\hat{u}(\nu) \varphi^{\prime} \leq-\hat{u}(\nu) \varphi+O(1)$ by definition, so $\sigma(u, \varphi) \geq-\hat{u}(\varphi)$. On the other hand, $\hat{\varphi}(\nu)=-1$, so $u \leq$ $\sigma(u, \varphi) \varphi+O(1)$ implies $-\hat{u}(\nu) \geq \sigma(u, \varphi)$ hence $-\hat{u}(\nu)=\sigma(u, \varphi)$.

Now if $f \in \mathcal{J}(t \varphi)$, then $f \in \mathcal{L}^{2}((t-\varepsilon) \hat{\varphi})$ for each $\varepsilon>0$ by Theorem 5.5 , hence $\log |f| \leq t \hat{\varphi}+A$. In particular, we get $\nu(f) \geq t-A(\nu)$. By definition of $\varphi$, this implies that $\log |f|$ is more singular than $(t-C) \varphi$ with $C:=A(\nu)$. Hence $\varphi$ is tame.

We finally prove a strong form of uniqueness: suppose we are given two maximal psh weights $\varphi, \varphi^{\prime}$ satisfying $\sigma(\log |f|, \varphi)=\sigma\left(\log |f|, \varphi^{\prime}\right)$ for all $f \in \mathcal{O}$. Both weights are tame and $\hat{\varphi}=\hat{\varphi}^{\prime}$ by what precedes, hence $\varphi=\varphi^{\prime}$ up to a bounded function by Theorem 5.12. This concludes the proof.

\section{§5.5. Valuations and generalized Lelong numbers}

Demailly [D1] defined the generalized Lelong number $\nu_{\varphi}(u)$ of a psh germ $u$ with respect to a psh weight $\varphi$ as the mass at the origin of the measure $d d^{c} u \wedge$ $\left(d d^{c} \varphi\right)^{n-1}$. The generalized Lelong number satisfies the following comparison principle: if $u$ is more singular than $u^{\prime}$ and $\varphi$ is more singular than $\varphi^{\prime}$, then $\nu_{\varphi}(u) \geq \nu_{\varphi^{\prime}}\left(u^{\prime}\right)$. We are now in position to relate the generalized Lelong number with respect to a tame weight to an average of valuations, largely by repeating the argument given in [FJ2].

Proof of Theorem B. Pick a tame psh weight $\varphi$ and any psh germ $u$. We first prove that $\hat{u}$ is integrable with respect to the Monge-Ampère measure $\operatorname{MA}(\hat{\varphi})$. Let $\varphi_{k}:=\frac{1}{k} \mathcal{R}(k \varphi)$. Since $\varphi$ is tame, there is a constant $C>0$ such that $\varphi \leq \varphi_{k} \leq(1-C / k) \varphi$ up to bounded terms. It follows that $Z(\varphi) \leq Z\left(\varphi_{k}\right) \leq$ $(1-C / k) Z(\varphi)$ for each $k$, and thus $\left\langle Z(u), Z(\varphi)^{n-1}\right\rangle \geq\left\langle Z(u), Z\left(\varphi_{k}\right)^{n-1}\right\rangle \geq$ 
$(1-C / k)^{n-1}\left\langle Z(u), Z(\varphi)^{n-1}\right\rangle$. Since $Z\left(\varphi_{k}\right)$ is a Cartier divisor, the MongeAmpère measure $\operatorname{MA}\left(\hat{\varphi}_{k}\right)$ is finite and $\int_{\mathcal{V}} \hat{u} \operatorname{MA}\left(\hat{\varphi}_{k}\right)>-\infty$. It thus follows that $\operatorname{MA}(\hat{\varphi})$ is also finite.

The previous argument actually shows $\int_{\mathcal{V}} \hat{u} \mathrm{MA}(\hat{\varphi})=\lim _{k} \int_{\mathcal{V}} \hat{u} \mathrm{MA}\left(\hat{\varphi}_{k}\right)$. On the other hand, by the comparison principle, it is clear that $\nu_{\varphi}(u) \geq$ $\nu_{\varphi_{k}}(u) \geq(1-C / k)^{n-1} \nu_{\varphi}(u)$, and in particular $\nu_{\varphi_{k}}(u)$ converges to $\nu_{\varphi}(u)$. We are thus reduced to proving the theorem when the weight $\varphi$ has singularities described by a primary ideal $\mathfrak{a}$.

Replacing $\mathfrak{a}$ by an ideal having the same integral closure changes the function $\varphi$ by a bounded term only. By choosing generic linear combination of a system of generators of $\mathfrak{a}$, we may assume that the ideal is generated by exactly $n$ holomorphic functions $f_{1}, \ldots, f_{n}$. Consider the map $F:\left(\mathbf{C}^{n}, 0\right) \rightarrow\left(\mathbf{C}^{n}, 0\right)$ given in coordinates by $F=\left(f_{1}, \cdots, f_{n}\right)$. As a is primary, $F$ is finite, and by construction $\varphi=\log |F|$.

Denote by $e(F, \cdot)$ the local topological degree of $F$. For any point $p$ near the origin, set $F_{*} u(p)=\sum_{F(q)=p} e(F, q) u(q)$. Then $F_{*} u$ is a psh germ, and we have

$$
\begin{aligned}
\nu_{\varphi}(u) & =\left(d d^{c} u \wedge\left(d d^{c} \log |F|\right)^{n-1}\right)\{0\} \\
& =\left(d d^{c}\left(F_{*} u\right) \wedge\left(d d^{c} \log |z|\right)^{n-1}\right)\{0\}=\nu^{\mathrm{L}}\left(F_{*} u\right) .
\end{aligned}
$$

Similarly if $f \in \mathcal{O}$, then $F_{*} f \in \mathcal{O}$ is defined by $F_{*} f(p)=\prod_{F(q)=p} f(q)^{e(F, q)}$. If $\mathfrak{a}$ is primary, then so is $F_{*} \mathfrak{a}$.

Next, if $u$ has singularities described by an ideal $\mathfrak{b}$, then $\hat{u}=\log |\mathfrak{b}|$, and $F_{*} u$ has singularities described by $F_{*} \mathfrak{b}$. By the projection formula in intersection theory and (4.3), we get

$$
\int_{\mathcal{V}}-\hat{u} \operatorname{MA}(\hat{\varphi})=\left\langle\mathfrak{b}, \mathfrak{a}^{\langle n-1\rangle}\right\rangle=\left\langle\mathfrak{b}, F^{*} \mathfrak{m}^{\langle n-1\rangle}\right\rangle=\left\langle F_{*} \mathfrak{b}, \mathfrak{m}^{\langle n-1\rangle}\right\rangle=\nu^{\mathrm{L}}\left(F_{*} u\right),
$$

which shows the formula in Theorem B in this case. To deal with a general psh germ, we rely again on the approximation technique of Demailly. Pick $\phi \in \mathcal{H}(u, B)$ with norm $\int_{B}|\phi|^{2} e^{-2 u}=1$. The mean value theorem yields for all points in the ball $B(r / 2)$ of radius $r / 2$ and centered at the origin:

$$
\left|F_{*} \phi\right|^{2} \leq \frac{1}{c_{n} r^{2 n}} \int_{B(r)}\left|F_{*} \phi\right|^{2} d \lambda \leq \frac{1}{c_{n} r^{2 n}} \sup _{B(r)} e^{2 F_{*} u} \cdot \int_{B(r)}\left|F_{*} \phi\right|^{2} e^{-2 F_{*} u} d \lambda .
$$


Now the change of variables formula gives

$$
\begin{aligned}
\int_{B(r)}\left|F_{*} \phi\right|^{2} e^{-2 F_{*} u} d \lambda & =e(F, 0) \cdot \int_{F^{-1} B(r)}|\phi|^{2} e^{-2 u}|J F|^{2} d \lambda \\
& \leq e(F, 0) \cdot \sup |J F|^{2} \cdot \int_{B}|\phi|^{2} e^{-2 u} d \lambda \leq C
\end{aligned}
$$

for some uniform constant $C>0$. Hence $\log \left|F_{*} \phi\right| \leq \sup _{B(r)} F_{*} u-C^{\prime} \log r$, and by definition of $\mathcal{R}(u)$ we $\operatorname{infer} \sup _{B(r / 2)} F_{*} \mathcal{R}(u) \leq \sup _{B(r)} F_{*} u-C^{\prime} \log r$. Set $u_{k}:=k^{-1} \mathcal{R}(k u)$. Then

$$
\nu^{\mathrm{L}}\left(F_{*} u_{k}\right) \leq \nu^{\mathrm{L}}\left(F_{*} u\right) \leq \nu^{\mathrm{L}}\left(F_{*} u_{k}\right)+\frac{C^{\prime \prime}}{k} .
$$

In particular we get a sequence of psh functions with singularities described by analytical ideals for which $\nu^{\mathrm{L}}\left(F_{*} u_{k}\right) \rightarrow \nu^{\mathrm{L}}\left(F_{*} u\right)$. We saw that $\hat{u}_{k} \rightarrow \hat{u}$ pointwise on $\mathcal{V}_{\mathrm{qm}}$. As $\operatorname{MA}(\hat{\varphi})$ is supported on finitely many divisorial valuations (Proposition 4.9), we infer $\int_{\mathcal{V}}-\hat{u}_{k} \operatorname{MA}(\hat{\varphi}) \rightarrow \int_{\mathcal{V}}-\hat{u} \operatorname{MA}(\hat{\varphi})$. Finally we conclude by applying (5.5) for each $u_{k}$ and letting $k \rightarrow \infty$.

\section{§5.6. Proof of Theorem A}

Consider two psh germs $u, v$. By construction, (1) is equivalent to $\hat{u}=\hat{v}$. Theorem 5.13 shows that (3) implies (1). Moreover, (1) implies (3) and (4) by Theorem 5.12 and Theorem B, respectively, and (1) implies (2) by Theorem 5.5. It remains to be shown that (2) implies (1). So suppose $\mathcal{J}_{+}(t u)=\mathcal{J}_{+}(t v)$ for all $t$. Theorem 5.5 yields $\mathcal{L}^{2}(t \hat{u}) \cap \mathcal{O}=\mathcal{L}^{2}(t \hat{v}) \cap \mathcal{O}$ for all $t$, but this does a priori not imply that $\mathcal{L}^{2}(t \hat{u})=\mathcal{L}^{2}(t \hat{v})$, so we cannot directly apply Proposition 3.13 to conclude $\hat{u}=\hat{v}$. However, (5.3) applied to $(1+\varepsilon) u$ shows that $\log \left|\mathcal{J}_{+}(u)\right| \geq$ $g_{u} \geq \log \left|\mathcal{J}_{+}(u)\right|-A$, and thus $\hat{u}$ is also the limit of $\frac{1}{k} \log \left|\mathcal{J}_{+}(k u)\right|$ as $k \rightarrow \infty$, so the result follows.

\section{References}

[A] P. Autissier, Points entiers sur les surfaces arithmétiques, J. Reine Angew. Math. 531 (2001), 201-235.

[BR] M. H. Baker and R. Rumely, Equidistribution of small points, rational dynamics, and potential theory, Ann. Inst. Fourier (Grenoble) 56 (2006), no. 3, 625-688.

[Be] V. G. Berkovich, Spectral theory and analytic geometry over non-Archimedean fields, 33, Amer. Math. Soc., Providence, RI, 1990.

[BGS] S. Bloch, H. Gillet and C. Soulé, Non-Archimedean Arakelov theory, J. Algebraic Geom. 4 (1995), no. 3, 427-485. 
[BT] E. Bedford and B. A. Taylor, A new capacity for plurisubharmonic functions, Acta Math. 149 (1982), no. 1-2, 1-40.

[Bo] J.-B. Bost, Potential theory and Lefschetz theorems for arithmetic surfaces, Ann. Sci. École Norm. Sup. (4) 32 (1999), no. 2, 241-312.

[BFJ] S. Boucksom, C. Favre and M. Jonsson, Differentiability of volumes of divisors and a problem of Teissier, www.arxiv.org/math.AG/0608260, to appear in J. Alg. Geom.

[CLR] T. Chinburg, C. F. Lau and R. Rumely, Capacity theory and arithmetic intersection theory, Duke Math. J. 117 (2003), no. 2, 229-285.

[Ch] A. Chambert-Loir, Mesures et équidistribution sur les espaces de Berkovich, J. Reine Angew. Math. 595 (2006), 215-235.

[Co] V. Cossart, Uniformisation et désingularisation des surfaces d'après Zariski, in Resolution of singularities (Obergurgl, 1997), 239-258, Progr. Math., 181, Birkhäuser, Basel.

[D1] J.-P. Demailly, Nombres de Lelong généralisés, théorèmes d'intégralité et d'analyticité, Acta Math. 159 (1987), no. 3-4, 153-169.

[D2] Monge-Ampère operators, Lelong numbers and intersection theory, in Complex analysis and geometry, 115-193, Plenum, New York, 1993.

[DEL] J.-P. Demailly, L. Ein and R. Lazarsfeld, A subadditivity property of multiplier ideals, Michigan Math. J. 48 (2000), 137-156.

[DK] J.-P. Demailly and J. Kollár, Semi-continuity of complex singularity exponents and Kähler-Einstein metrics on Fano orbifolds, Ann. Sci. École Norm. Sup. (4) 34 (2001), no. 4, 525-556.

[ELS] L. Ein, R. Lazarsfeld and K. E. Smith, Uniform approximation of Abhyankar valuation ideals in smooth function fields, Amer. J. Math. 125 (2003), no. 2, 409-440.

[FJ1] C. Favre and M. Jonsson, The valuative tree, Lecture Notes in Math. 1853, Springer, Berlin, 2004.

[FJ2] (2005), no. 2, 271-311.

[FJ3] Valuations and multiplier ideals, J. Amer. Math. Soc. 18 (2005), no. 3, 655-684 (electronic).

[FJ4] Eigenvaluations, Ann. Sci. École Norm. Sup. (4) 40 (2007), no. 2, 309-349.

[G] W. Gubler, Local heights of subvarieties over non-Archimedean fields, J. Reine Angew. Math. 498 (1998), 61-113.

[Hi] H. Hironaka, Resolution of singularities of an algebraic variety over a field of characteristic zero. I, II, Ann. of Math. (2) 79 (1964), 109-203; ibid. (2) 79 (1964), $205-326$.

[Ho] J. A. Howald, Multiplier ideals of monomial ideals, Trans. Amer. Math. Soc. 353 (2001), no. 7, 2665-2671 (electronic).

[Hö] L. Hörmander, Notions of convexity, Progr. Math., 127, Birkhäuser Boston, Boston, MA, 1994.

[I] S. Izumi, A measure of integrity for local analytic algebras, Publ. Res. Inst. Math. Sci. 21 (1985), no. 4, 719-735.

[K] E. Kani, Potential theory on curves, in Théorie des nombres (Quebec, PQ, 1987), 475-543, de Gruyter, Berlin.

[KKMS] G. Kempf, F. F. Knudsen, D. Mumford and B. Saint-Donat, Toroidal embeddings. I, Lecture Notes in Math., 339, Springer, Berlin, 1973.

[Ki] C. O. Kiselman, Un nombre de Lelong raffiné, Séminaire d'analyse complexe et géométrie 1985-1987, 61-70, Faculté des sciences de Tunis et Faculté des sciences et techniques de Monastir.

[Ko] J. Kollár, Singularities of pairs, in Algebraic geometry-Santa Cruz 1995, 221-287, Proc. Sympos. Pure Math., Part 1, Amer. Math. Soc., Providence, RI.

[KS] M. Kontsevich and Y. Soibelman, Affine structures and non-Archimedean analytic spaces, in The unity of mathematics, 321-385, Progr. Math., 244, Birkhäuser, 
Boston, Boston, MA.

[L] R. Lazarsfeld, Positivity in algebraic geometry. II, Ergebnisse der Mathematik und ihrer Grenzgebiete, 49, Springer, Berlin, 2004.

[M] V. Maillot, Géométrie d'Arakelov des variétés toriques et fibrés en droites intégrables, Mém. Soc. Math. Fr. (N.S.) No. 80 (2000), vi+129 pp.

[Ram] C. P. Ramanujam, On a geometric interpretation of multiplicity, Invent. Math. 22 (1973/74), 63-67.

[Ras1] A. Rashkovskii, Relative types and extremal problems for plurisubharmonic functions, Int. Math. Res. Not. 2006 (2006), Art. ID 76283, 26 pp.

[Ras2] , Indicators for plurisubharmonic functions of logarithmic growth, Indiana Univ. Math. J. 50 (2001), no. 3, 1433-1446.

[Re] D. Rees, Generalizations of reductions and mixed multiplicities, J. London Math. Soc. (2) 29 (1984), no. 3, 397-414.

[S] V. V. Shokurov, Prelimiting flips, Tr. Mat. Inst. Steklova 240 (2003), Biratsion. Geom. Linein. Sist. Konechno Porozhdennye Algebry, 82-219; translation in Proc. Steklov Inst. Math. 2003, no. 1 (240), 75-213.

[T1] A. Thuillier, Théorie du potentiel sur les courbes en géométrie analytique non archimédienne. Applications à la théorie d'Arakelov, Ph.D. Thesis, Université de Rennes I.

[T2] — Géométrie toroïdale et géométrie analytique non archimédienne. Application au type d'homotopie de certains schémas formels, Manuscripta Math. 123 (2007), no. 4, 381-451.

[V] M. Vaquié, Valuations, in Resolution of singularities (Obergurgl, 1997), 539-590, Progr. Math., 181, Birkhäuser, Basel.

[Z] O. Zariski, The compactness of the Riemann manifold of an abstract field of algebraic functions, Bull. Amer. Math. Soc. 50 (1944), 683-691.

[ZS] O. Zariski and P. Samuel, Commutative algebra, Vol. II, Reprint of the 1960 edition, Springer, New York, 1975.

[Zh] S. Zhang, Positive line bundles on arithmetic varieties, J. Amer. Math. Soc. 8 (1995), no. 1, 187-221. 